\title{
ON RIGIDITY, ORIENTABILITY AND CORES OF RANDOM GRAPHS WITH SLIDERS
}

\author{
J. BARRÉ ${ }^{1}$, \\ M. LELARGE ${ }^{2}$, \\ D. MITSCHE ${ }^{1}$
}

\begin{abstract}
Suppose that you add rigid bars between points in the plane, and suppose that a constant fraction $q$ of the points moves freely in the whole plane; the remaining fraction is constrained to move on fixed lines called sliders. When does a giant rigid cluster emerge? Under a genericity condition, the answer only depends on the graph formed by the points (vertices) and the bars (edges). We find for the random graph $G \in \mathcal{G}(n, c / n)$ the threshold value of $c$ for the appearance of a linear-sized rigid component as a function of $q$, generalizing results of 7 . We show that this appearance of a giant component undergoes a continuous transition for $q \leq 1 / 2$ and a discontinuous transition for $q>1 / 2$. In our proofs, we introduce a generalized notion of orientability interpolating between 1- and 2-orientability, of cores interpolating between 2core and 3-core, and of extended cores interpolating between $2+1$-core and $3+2$-core; we find the precise expressions for the respective thresholds and the sizes of the different cores above the threshold. In particular, this proves a conjecture of 7 about the size of the $3+2$-core. We also derive some structural properties of rigidity with sliders (matroid and decomposition into components) which can be of independent interest.
\end{abstract}

\section{INTRODUCTION}

Consider a set of points, some of them allowed to move freely in the Euclidean plane, and some constrained to move on fixed lines, called sliders. The free points have two degrees of freedom, the points attached to sliders have only one. Now, add bars between pairs of these points; a bar fixes the length between the two endpoints. The points and bars form a framework. A framework is said to be rigid if it cannot be deformed (but can possibly be translated and rotated on the plane); equivalently, it is rigid if the distance between any pair of points, connected by a bar or not, is fixed. Characterizing the rigidity of a framework is very difficult in general. In the absence of sliders, a celebrated theorem by Laman [8] ensures that for a generic framework, its rigidity properties only depend on its underlying graph, where points are vertices and bars are edges: the geometry does not enter. This theorem has been generalized to frameworks with sliders in [18. In the whole article, we will implicitly assume that all frameworks are generic, so that rigidity has a purely graph theoretical characterization and we can deal with vertices and edges instead of points and bars. This will be detailed in Section 2 .

\footnotetext{
${ }^{1}$ Laboratoire J. A. Dieudonné, UMR CNRS 7351, Université de Nice-Sophia Antipolis, Parc Valrose, 06108 Nice France, email: \{julien.barre, dmitsche\}@unice.fr

${ }^{2}$ INRIA-ENS, Paris, France, email: marc.lelarge@inria.fr

Julien Barré thanks Victor Mizrahi and Alain Olivetti for many discussions.
} 
We will call a vertex of type 1 (resp. type 2) if it is (resp. is not) connected to a slider. Consider now a percolation setting: take a set of $n$ vertices, a fraction $q$ of which are type 2 , and add edges randomly. The questions are: When does a giant (that is: including a positive fraction of the vertices) rigid structure emerge? What is its size? When edges are sampled independently at random between pairs of vertices, the resulting graph is an Erdős-Rényi random graph $G(n, c / n)$. In this case and for $q=1$ (no slider), Kasiviswanathan et al. 7] showed that the threshold for a giant rigid component is $c \simeq 3.588$, and that the transition is discontinuous: as soon as the giant rigid component appears, it already includes a positive fraction of all $n$ vertices. This recovers numerical and heuristic results found earlier in the physics literature [14, 4, and contrasts with the emergence of a giant connected component at $c=1$, which is continuous.

Indeed when $q=0$, we will see that rigidity is closely related to the emergence of the giant connected component. Our goal is to investigate the case where $q \in[0,1]$. We are thus interested in situations interpolating between standard connectivity percolation and rigidity percolation as studied in [7]. We obtain the following results:

- We compute the threshold for rigidity percolation as a function of $q$

- We show that the transition is continuous for $q \leq 1 / 2$ and discontinuous for $q>1 / 2$, thus uncovering what is called a "tricritical" point in statistical mechanics, for $q=1 / 2$

- On the way, we obtain new results on cores for Erdős-Rényi random graphs and their generalization to two types of vertices. We prove in particular a conjecture on the size of the $3+2$-core in 7

Rigidity percolation has physical motivations: it is a model to understand some properties of network glasses and proteins [20, 16, 3, 17]. Thus, problems related to ours have been investigated by theoretical physicists. We have already cited investigations on random graphs starting with [14, 4, with only one type of vertex (type 2, or more generally type $k$ ). In 13 , Moukarzel heuristically studies a model with two types of vertices: a fraction of the vertices are pinned to the plane, instead of being allowed to move in one direction; they could be called "type 0" vertices. In this case, the transition disappears when the fraction of pinned vertices increases: there is no tricritical point, but rather a critical point.

In order to compute the threshold for rigidity, we use the same connection as [7] between orientability and rigidity. We then use recently introduced and powerful methods to compute the orientability threshold [11. To investigate the continuous or discontinuous character of the transition, we rely on various refinements of a method introduced in [6] to investigate the cores of a random graph. In Section 2 , we define our notion of rigidity with sliders and state our main results for ErdösRényi random graphs. In Section 3, we gather our structural results for rigidity with sliders: matroid and decomposition into components. We then prove our results for random graphs: in Section 4, we compute the orientability threshold, in Section 5. we relate it to rigidity. We then prove our main Theorems in Sections 6, 7 and 8. Finally a technical but important Lemma is proved in Section 9 .

\section{Some DEFinitions on Rigidity AND STATEMENTS OF RESUlts}

Throughout this paper log denotes the natural logarithm. Also, throughout the paper $G$ is a graph $(V, E)$ with $|V|=n$ and $|E|=m$. All our graphs are simple. 
Vertices are either of type 1 or of type 2 , and for $i \in\{1,2\}, n_{i}$ denotes the number of vertices of type $i$, so that $n=n_{1}+n_{2}$.

Subgraphs are typically denoted by $G^{\prime}$ with $n_{i}\left(G^{\prime}\right)$ vertices of type $i \in\{1,2\}$, $n\left(G^{\prime}\right)=n_{1}\left(G^{\prime}\right)+n_{2}\left(G^{\prime}\right)$ vertices in total and $m\left(G^{\prime}\right)$ edges. When the context is clear, we use the notations: $n^{\prime}=n\left(G^{\prime}\right), n_{i}^{\prime}=n_{i}\left(G^{\prime}\right)$ and $m^{\prime}=m\left(G^{\prime}\right)$.

Definition 2.1. Let $G$ be a graph with $n=n_{1}+n_{2}$ vertices and $m$ edges. $G$ is sparse if for all subgraph $G^{\prime} \subseteq G$ on $n^{\prime}=n_{1}^{\prime}+n_{2}^{\prime} \geq 2$ vertices and $m^{\prime}$ edges, we have:

$$
m^{\prime} \leq n_{1}^{\prime}+2 n_{2}^{\prime}+\min \left(0, n_{1}^{\prime}-3\right)=2 n^{\prime}-\max \left(n_{1}^{\prime}, 3\right) .
$$

In terms of physics, a sparse graph represents a structure without redundant constraint. The special treatment needed for subgraphs with 0,1 or 2 vertices of type 1 , i.e. when $n_{1}^{\prime}<3$, can then be understood: a structure which is not connected at all to the underlying plane (that is $n_{1}^{\prime}=0$ ) cannot be pinned, and always keeps at least three degrees of freedom, hence the -3 ; a structure with one slider (that is $n_{1}^{\prime}=1$ ) always keeps at least two degrees of freedom, hence the -2 ; and similarly for $n_{1}^{\prime}=2$. If $n_{1}^{\prime} \geq 3$, the structure can be completely pinned to the underlying plane, and thus has zero degrees of freedom.

Remark 2.2. We follow here Streinu and Theran [18, with a simplified terminology to make the present article easier to read. The present definition of sparsity corresponds to their $(2,0,3)$-graded-sparsity, for a restricted class of graphs (they consider also multiple graphs, and more types of vertices). Since we are only using two concepts of sparsity (see definition of Laman-sparsity below), no confusion should arise. To make the connection more explicit, note that our "type 1 vertices" correspond to vertices "with one attached loop" in [18].

We recall the standard definition:

Definition 2.3. $G$ is Laman-sparse if for all subgraph $G^{\prime} \subseteq G$ with $n^{\prime} \geq 2, m^{\prime} \leq$ $2 n^{\prime}-3$.

Laman-sparsity and sparsity are equivalent if there are only vertices of type 2 , i.e. $n=n_{2}$. Moreover a sparse graph is always Laman-sparse.

Definition 2.4. $G$ is minimally rigid if either $n=1$, or $G$ is sparse and

$$
m=n_{1}+2 n_{2}+\min \left(0, n_{1}-3\right) .
$$

Lemma 2.5. If $G$ is minimally rigid with $n_{1}<6$, then $G$ is connected.

Proof. Consider a partition of the vertices in two parts with $n_{a}$ and $n_{b}$ vertices respectively. Let $m_{a}$ and $m_{b}$ be the number of edges induced by each part. By the sparsity of $G$, we have $m_{i} \leq 2 n_{i}-3$ for $i \in\{a, b\}$. Hence, we have

$$
m-\left(m_{a}+m_{b}\right) \geq 2 n-\max \left(3, n_{1}\right)-2 n_{a}-2 n_{b}+6=6-\max \left(3, n_{1}\right),
$$

so that for $n_{1}<6$, the two parts are connected.

Remark 2.6. For $n_{1} \geq 6$, a minimally rigid graph $G$ does not need to be connected as seen by considering the disjoint union of two cliques of size three with all nodes of type 1 . 
Remark 2.7. Streinu and Theran (see [18) use a slightly different definition of rigidity. In our notation, for them $G$ is minimally rigid if $G$ is sparse and $m=n_{1}+$ $2 n_{2}$. This definition is not equivalent to ours: using our Definition 2.4. physically, it means that we consider as rigid a structure that cannot be deformed (but can possibly be moved over the plane as a solid object). Streinu and Theran (see [18]) consider as rigid a structure that cannot be deformed, and that is pinned on the plane; in particular, rigidity in this sense implies $n_{1} \geq 3$. Definition 2.4, however, coincides with the standard definition of rigidity when there are only vertices of type 2, and this will be convenient to compare our results with the results of [7; it will also allow us to use some of their results.

Recall that a spanning subgraph is one that includes the entire vertex set $V$.

Definition 2.8. A graph is rigid if it contains a spanning subgraph which is minimally rigid. A rigid block in $G$ is defined to be a vertex-induced rigid subgraph. $A$ rigid component of $G$ is an inclusion-wise maximal block.

Remark 2.9. Note that for a sparse graph $G$, a rigid block is always minimally rigid.

Note that a rigid component does not need to be connected. By definition, it is clear that rigidity is preserved under addition of edges and that the size of the largest (in terms of vertices covered) rigid component of a graph can only increase when edges are added.

We now describe our probabilistic setting: consider for the following statements the random graph $G \in \mathcal{G}(n, c / n)$ where each edge is present independently with probability $c / n$, with $c>0$. For such a graph we also write $G(n, c / n)$ below. Each vertex gets type 1 with probability $1-q$ and type 2 with probability $q$, where $q \in[0,1]$.

To state our result, we need some notations. Let $Q(x, y)=e^{-x} \sum_{j \geq y} \frac{x^{j}}{j !}$. We define the function $c^{*}(q)$ as follows:

- for $q \leq 1 / 2$, we set $c^{*}(q)=\frac{1}{1-q}$;

- for $q>1 / 2$, let $\xi^{*}=\xi^{*}(q)$ be the positive solution to:

$$
\xi \frac{(1-q) Q(\xi, 1)+q Q(\xi, 2)}{(1-q) Q(\xi, 2)+2 q Q(\xi, 3)}=2 .
$$

In this case we set:

$$
c^{*}(q)=\frac{\xi^{*}}{(1-q) Q\left(\xi^{*}, 1\right)+q Q\left(\xi^{*}, 2\right)} .
$$

It will follow from the proof that the equation for $\xi^{*}$ has indeed a unique positive solution and that for $q>1 / 2, c^{*}(q)<\frac{1}{1-q}$.

We can now state our first theorem:

Theorem 2.10. Let $G=G(n, c / n)$ with $c>0$, and let $q \in[0,1]$. Let $R_{n}(q, c)$ $\left(R_{n}^{C}(q, c)\right.$, resp.) be the number of vertices covered by the largest rigid component (connected rigid block, resp.) of $G$.

- For $c>c^{*}(q)$, there is a giant rigid component in $G$, i.e., there exists $\alpha=\alpha(q, c)>0$ such that

$$
\mathbb{P}\left(\frac{R_{n}(q, c)}{n} \geq \alpha\right) \rightarrow 1 \text { when } n \rightarrow \infty
$$


ON RIGIDITY, ORIENTABILITY AND CORES OF RANDOM GRAPHS WITH SLIDERS 5

- For $c<c^{*}(q)$, there is no giant rigid component in $G$; i.e.,

$$
\forall \alpha>0, \mathbb{P}\left(\frac{R_{n}(q, c)}{n} \geq \alpha\right) \rightarrow 0 \text { when } n \rightarrow \infty
$$

The above results also hold true for $R_{n}^{C}(q, c)$. Moreover, for $c>c^{*}(q)$, a.a.s., there is one unique giant rigid component (one unique giant connected rigid block, resp.).

Our next theorem states that the transition as $c$ varies and $q$ is held fixed is continuous for $q \leq 1 / 2$ and discontinuous for $q>1 / 2$. More precisely, we have the following:

Theorem 2.11. - The transition is discontinuous for $q>1 / 2$ : let $q>1 / 2$; there is $\alpha(q)=\alpha>0$ such that for any $c>c^{*}(q)$

$$
\lim _{n \rightarrow \infty} \mathbb{P}\left(\frac{R_{n}(q, c)}{n} \geq \alpha\right)=1
$$

- The transition is continuous for $q \leq 1 / 2$ : let $q \leq 1 / 2$; for any $\alpha>0$,

$$
\lim _{c \rightarrow \frac{1}{1-q}} \lim _{n \rightarrow \infty} \mathbb{P}\left(\frac{R_{n}(q, c)}{n} \geq \alpha\right)=0
$$

We now relate rigidity and orientability. We start with the following definition of 1.5-orientability.

Definition 2.12. A graph is 1.5-orientable if there exists an orientation of the edges such that type 1 vertices have in-degree at most 1 and type 2 vertices have in-degree at most 2.

A standard argument in the context of network flows gives (see Proposition 3.3 in 15 )

Proposition 2.13. A graph $G$ is 1.5-orientable if and only if for every induced subgraph $G^{\prime}$ of $G, m^{\prime} \leq n_{1}^{\prime}+2 n_{2}^{\prime}$.

As a corollary, we see that a sparse graph $G$ is always 1.5-orientable. Moreover, we see that if $G$ is 1.5 -orientable, then $G$ will remain 1.5 -orientable after removing some edges and if $G$ is not 1.5-orientable then adding edges cannot make it 1.5orientable.

Our next theorem shows that the threshold for being 1.5-orientable for the random graph $G(n, c / n)$ is the same as the one for the appearance of a giant rigid component.

Theorem 2.14. Let $G=G(n, c / n)$ with $c>0$, and let $q \in[0,1]$.

(a) if $c<c^{*}(q), G$ is 1.5-orientable a.a.s.

(b) if $c>c^{*}(q), G$ is not 1.5-orientable a.a.s.

We now relate the notion of rigidity and 1.5-orientability with a new notion of core.

Definition 2.15. For a graph with type 1 and type 2 vertices, the 2.5-core is the largest induced subgraph with all type 1 vertices with degree at least 2 and all type 2 vertices with degree at least 3. 
Note that this definition coincides with the 2-core (3-core, resp.) if the graph contains only type 1 vertices (type 2 , resp.).

One can show that we can construct the 2.5-core by removing recursively type 1 vertices with degree at most 1 and type 2 vertices with degree at most 2 . Note that the 2.5-core can be empty and in this case, the graph is 1.5 -orientable. More generally, a graph $G$ is 1.5 -orientable if and only if its 2.5 -core is orientable.

Clearly the size of the 2.5-core can only increase with the addition of edges. In our probabilistic setting, it turns out that for a fixed $q$, the 2.5-core appears at a value $\tilde{c}(q) \leq c^{*}(q)$.

Let $Q(x, y)$ as before. We define

$$
\tilde{c}(q)=\inf _{\xi>0} \frac{\xi}{(1-q) Q(\xi, 1)+q Q(\xi, 2)} .
$$

Note that when $\xi \rightarrow 0$, we have $\frac{\xi}{(1-q) Q(\xi, 1)+q Q(\xi, 2)} \rightarrow \frac{1}{1-q}$, in particular $\tilde{c}(q) \leq \frac{1}{1-q}$. Let $\tilde{\xi}(q, c)$ be the largest solution to

$$
\xi=c(1-q) Q(\xi, 1)+c q Q(\xi, 2) .
$$

We can now state the theorem:

Theorem 2.16. Let $G=G(n, c / n)$ with $c>0$ and let $q \in[0,1]$. Let Core be the 2.5-core of $G, n_{1}$ (Core) ( $n_{2}$ (Core), resp.) be the number of nodes of type 1 (type 2, resp.) in the core and $m$ (Core) be the number of edges in the core. We have

(a) if $c<\tilde{c}(q)$ and $q>0$, then a.a.s. the 2.5-core has $o_{p}(n)$ vertices.

(b) if $c>\tilde{c}(q)$, then a.a.s. the 2.5-core is such that $n_{1}$ (Core) $/ n \rightarrow(1-$ q) $Q(\tilde{\xi}(q, c)), 2), n_{2}($ Core $\left.) / n \rightarrow q Q(\tilde{\xi}(q, c)), 3\right)$, and $2 m($ Core $) / n \rightarrow \tilde{\xi}(q, c)((1-q) Q(\tilde{\xi}(q, c)), 1)+q Q(\tilde{\xi}(q, c)), 2))$.

Remark 2.17. When the core is not $o_{p}(n)$, i.e., when $c>\tilde{c}(q)$, we have

$$
\frac{m(\text { Core })}{n_{1}(\text { Core })+2 n_{2}(\text { Core })} \rightarrow \frac{\tilde{\xi}(q)}{2} \frac{(1-q) Q(\tilde{\xi}(q)), 1)+q Q(\tilde{\xi}(q)), 2)}{(1-q) Q(\tilde{\xi}(q)), 2)+2 q Q(\tilde{\xi}(q)), 3)} .
$$

In particular, if this ratio is larger than one, then the 2.5-core is not 1.5-orientable. A simple computation shows that this ratio becomes larger than one exactly for $c>$ $c^{*}(q) \geq \tilde{c}(q)$. Moreover, we have $c^{*}(q)=\tilde{c}(q)=\frac{1}{1-q}$ for $q \leq 0.5$ and $c^{*}(q)>\tilde{c}(q)$ for $q>0.5$.

Remark 2.18. When $q$ is fixed and we increase $c$ from 0 to infinity, it is easy to note the following from previous theorem: when $q \leq 1 / 2$, the size of the 2.5-core is continuous in $c$ whereas for $q>1 / 2$, the 2.5-core appears discontinuously.

In the absence of sliders $(q=0)$, the largest rigid component is closely related to the $3+2$-core [7. This led the authors of [7] to formulate a conjecture on the size of the $3+2$-core. We introduce now a generalization of the $3+2$-core which will play a role in our proof of Theorem 2.11.

Definition 2.19. Starting from the 2.5-core, one constructs a larger subgraph as follows: add recursively type 1 vertices which are linked by one edge with the current subgraph, and type 2 vertices which are linked by two edges with the current subgraph. The resulting subgraph is called the $2.5+1.5$-core. 
Note that this definition coincides with the 2+1-core $(3+2$-core, resp. $)$ if the graph contains only type 1 vertices (type 2, resp.).

Furthermore, we also compute the threshold and the size of the 2.5+1.5-core. This proves a conjecture in 7 on the $3+2$-core. The proof follows again the ideas in $[6]$. We use the same definitions of $\tilde{c}(q)$ and $\tilde{\xi}(q)$ as before and state the following theorem:

Theorem 2.20. Let $G=G(n, c / n)$ with $c>0$ and $q \in[0,1]$. Let Core + be the $2.5+1.5$-core of $G$, and $n$ (Core +$)$ the number of vertices inside the $2.5+1.5$-core. If $c>\tilde{c}(q)$, where $\tilde{c}(q)$ is defined by (2), then a.a.s., $n($ Core +$) / n \rightarrow 1-e^{-\tilde{\xi}}-q \tilde{\xi} e^{-\tilde{\xi}}$, where $\tilde{\xi}$ is defined in (3).

Remark 2.21. For $q \leq 1 / 2$, we have $\tilde{c}(q)=\frac{1}{1-q}$, and if $c \searrow \frac{1}{1-q}$, then we have $\tilde{\xi} \rightarrow 0$, and thus $n($ Core +$) / n \rightarrow 0$.

For the proof of the aforementioned theorems, the following lemma plays a crucial role, and hence we state it already here: for a subgraph of size $n^{\prime}$, let $n_{1}^{\prime}$ its number of vertices of type 1 and $n_{2}^{\prime}$ its number of vertices of type 2 (we do not explicitly refer to the size nor to the subgraph, since it is clear from the context). Let $X_{n^{\prime}}$ denote the number of subgraphs of size $n^{\prime}$ with more than $n_{1}^{\prime}+2 n_{2}^{\prime}$ edges. We have:

Lemma 2.22. Let $q \in(0,1)$, and let $G \in \mathcal{G}(n, p)$ with $p=c / n$ and $c<\frac{1}{1-q}$. A.a.s., there exists a strictly positive constant $\alpha=\alpha\left(q, c-\frac{1}{1-q}\right)>0$ such that $\sum_{1 \leq n^{\prime} \leq \alpha n} X_{n^{\prime}}=0$.

Remark 2.23. Lemma 2.22 will also play the role of the Lemma 4.1 in [5], or Proposition 3.3 in [7. Lemma 4.1 in [5] ensures that all subgraphs of size u, with $m$ edges, such that $m / u>c_{1}>1$ are of size at least $\gamma n$ for some $\gamma>0$. In our case however, if $n_{2}$, the number of type 2 sites is much smaller than $n_{1}$, this lemma cannot be used. Lemma 2.22 provides the necessary refinement.

\section{Properties of (Deterministic) Sparse Graphs}

We gather in this section a few properties valid for general graphs, independently of the probabilistic setting. They will be useful later.

Given two subgraphs $A=\left(V_{A}, E_{A}\right)$ and $B=\left(V_{B}, E_{B}\right)$ of $G$, we denote by $A \cup B$ $(A \cap B$, resp. $)$ the subgraph of $G$ with vertex set $V_{A} \cup V_{B}\left(V_{A} \cap V_{B}\right.$, resp.) and edge set $E_{A} \cup E_{B}\left(E_{A} \cap E_{B}\right.$, resp.).

Lemma 3.1. Given two rigid blocks $A=\left(V_{A}, E_{A}\right)$ and $B=\left(V_{B}, E_{B}\right)$ of a sparse graph $G$, we have

- if $n(A \cap B) \geq 2$, then $A \cup B$ and $A \cap B$ are rigid blocks;

- if $n(A \cap B) \geq 1$ and $\min \left(n_{1}(A), n_{1}(B)\right) \geq 3$, then $n_{1}(A \cap B) \geq 3$ and in particular $A \cup B$ and $A \cap B$ are rigid blocks.

Proof. We first note that for any $x, y, z \geq 0$, such that $\min (x, y) \geq z$, we have

$$
\max (x+y-z, 3)+\max (z, 3) \geq \max (x, 3)+\max (y, 3) .
$$

Denoting by $m(\Delta)$ the number of edges between $V_{A} \backslash V_{B}$ and $V_{B} \backslash V_{A}$, we have

$$
\begin{aligned}
m(A \cup B) & =m(A)+m(B)-m(A \cap B)+m(\Delta) \\
& =2 n(A)-\max \left(n_{1}(A), 3\right)+2 n(B)-\max \left(n_{1}(B), 3\right)-m(A \cap B)+m(\Delta)
\end{aligned}
$$


By the sparsity of $G$, we have $m(A \cup B) \leq 2 n(A \cup B)-\max \left(n_{1}(A \cup B), 3\right)$, so that we get

$m(A \cap B) \geq 2 n(A \cap B)-\max \left(n_{1}(A), 3\right)-\max \left(n_{1}(B), 3\right)+\max \left(n_{1}(A \cup B), 3\right)+m(\Delta)$.

Using (4), we get

$$
m(A \cap B) \geq 2 n(A \cap B)-\max \left(n_{1}(A \cap B), 3\right)+m(\Delta) .
$$

First assume that $n(A \cap B) \geq 2$, so that by sparsity of $G$, we get $m(\Delta)=0$ and

$$
m(A \cap B)=2 n(A \cap B)-\max \left(n_{1}(A \cap B), 3\right) .
$$

Hence, we have

$$
\begin{aligned}
m(A \cup B)= & 2 n(A)-\max \left(n_{1}(A), 3\right)+2 n(B)-\max \left(n_{1}(B), 3\right) \\
& -2 n(A \cap B)+\max \left(n_{1}(A \cap B), 3\right) \\
\geq & 2 n(A \cup B)-\max \left(n_{1}(A \cup B), 3\right),
\end{aligned}
$$

so that by sparsity of $G$, we have indeed an equality and we proved the first point.

We now assume that $n(A \cap B) \geq 1$ and $\min \left(n_{1}(A), n_{1}(B)\right) \geq 3$, so that we have $m(A \cup B) \leq 2 n_{2}(A \cup B)+n_{1}(A \cup B)$ and then

$$
m(A \cap B) \geq 2 n_{2}(A \cap B)+n_{1}(A \cap B)+m(\Delta) .
$$

We see that $m(A \cap B) \geq 1$ and hence $n(A \cap B) \geq 2$. So, again by sparsity of $G$, we get

$$
m(A \cap B) \leq 2 n_{2}(A \cap B)+n_{1}(A \cap B)+\min \left(0, n_{1}(A \cap B)-3\right) .
$$

In particular, we have $n_{1}(A \cap B) \geq 3$ and then the second point follows from the first one.

Next, we show that by changing one vertex from type 2 to type 1 , a rigid graph remains rigid. This is the content of the following lemma:

Lemma 3.2. Let $G$ be a minimally rigid graph, and let $v$ be a type 2 vertex. Define $\tilde{G}$ as the same as $G$ where $v$ is transformed into a type 1 vertex. Then $\tilde{G}$ is rigid.

Proof. Assume first that $n_{1}(G)<3$. Then $\tilde{G}$ is actually even minimally rigid, and the statement follows: indeed, consider a subgraph $\tilde{H}$ of $\tilde{G}$. If $v \notin \tilde{H}$, the sparsity condition for $\tilde{H}$ is directly inherited from the sparsity of $G$. If $v \in \tilde{H}$, consider $H$ the subgraph of $G$ with the same vertices as $\tilde{H}$, except that $v$ is type 2 . Then from the sparsity of $G$, we have

$$
m(H) \leq 2 n(H)-3 \text {, and } m(H) \leq n_{1}(H)+2 n_{2}(H) .
$$

Since $n_{1}(H)<3$, it is enough to consider only the condition $m(H) \leq 2 n(H)-3$. Since $m(\tilde{H})=m(H), n(\tilde{H})=n(H)$, the condition $m(\tilde{H}) \leq 2 n(\tilde{H})-3$ is true, and since $n_{1}(\tilde{H}) \leq 3$, this condition is enough to ensure that $\tilde{H}$ verifies the sparsity condition. Hence $\tilde{G}$ is sparse. It is also clear that $\tilde{G}$ has exactly the right number of edges (we use again here $n_{1}(\tilde{G}) \leq 3$ ). Thus $\tilde{G}$ is rigid.

Assume now $n_{1}(G) \geq 3$. Now $\tilde{G}$ cannot be minimally rigid since it has one excess edge. We have to remove an edge, and the difficulty is to remove the right one. Define $H$ to be the smallest subgraph of $G$ such that:

- $H$ contains $v$

- $n_{1}(H) \geq 3$

- $H$ is minimally rigid 
$H$ exists since $G$ itself verifies all three conditions above. By Lemma 3.1, $H$ is unique and can be defined as the intersection of all subgraphs of $G$ verifying the above conditions. In particular, for any $K \subseteq G$ satisfying the above conditions, $H \subseteq K$.

Choose now $e$ any edge in $H$ and define $\bar{G}$ as follows:

$$
\bar{G}=\tilde{G} \backslash\{e\}
$$

We prove now that $\bar{G}$ is minimally rigid, which is clearly enough to show that $G$ is rigid: first, notice that $n_{1}(\bar{G})=n_{1}(G)+1 \geq 3$; with respect to $G$, one edge is removed and one vertex is turned from type 2 to type 1 , hence the total number of edges is correct. It thus remains to prove that $\bar{G}$ is sparse. Assume $\bar{G}$ is not sparse, and take a subgraph $K$ of $\bar{G}$ violating the sparsity condition. Note that if before changing $v$ from type 2 to type 1 , we have $n_{1}(K)<3$, by the argument at the beginning of the lemma, $K$ remains sparse, so we may assume that already in $G$ we have $n_{1}(K) \geq 3$. We have a few cases:

Case 1: If $v \notin K, K$ can be seen as a subgraph of $G$; the sparsity of $G$ implies that the sparsity condition for $K$ is true. This contradicts the hypothesis on $K$. Case 2: Assume now $v \in K$. We have, by assumption on $K$ not being sparse,

$$
m(K)=n_{1}(K)+2 n_{2}(K)+1 .
$$

Case 2a: Assume $H \subseteq K$. Let $K^{\prime}$ be the subgraph corresponding to the vertex set $K$ in $G$. $K^{\prime}$ had at most $n_{1}\left(K^{\prime}\right)+2 n_{2}\left(K^{\prime}\right)$ edges. Now, $v$ changed its type, but also one edge $e \in H$ has been removed, hence we have $m(K) \leq n_{1}(K)+2 n_{2}(K)$, and $K$ cannot violate sparsity.

Case 2b: Assume $H \nsubseteq K$. Define now $K^{\prime}$ to be equal to $K$, but turning vertex $v$ from type 1 back to type $2 . K^{\prime}$ is a subgraph of $G$. $G$ is sparse, hence $K^{\prime}$ is sparse. Since $n_{1}\left(K^{\prime}\right) \geq 3$ and since $m(K)=n_{1}(K)+2 n_{2}(K)+1$, we have

$$
m\left(K^{\prime}\right)=n_{1}\left(K^{\prime}\right)+2 n_{2}\left(K^{\prime}\right) .
$$

Hence $K^{\prime}$ is minimally rigid (in $G$ ). Also, $v \in K^{\prime}$, hence $K^{\prime}$ satisfies all properties defining $H$, and thus, by minimality $H \subseteq K^{\prime}$. This implies in turn that in $\tilde{G}$, $H \subseteq K$, which is a contradiction, finishing the proof.

The following proposition is closely related to the concept of "graded-sparsity matroids" introduced in [10. It shows that the matroid structure is retained within our slightly modified definitions.

Proposition 3.3. The collection of all minimally rigid graphs on $n_{1}$ vertices of type 1 and $n_{2}$ vertices of type 2 is the set of bases of a matroid whose ground set is the set of edges of the complete graph on $n=n_{1}+n_{2} \geq 2$ vertices.

Proof. The case $n_{1}=0$ is well-known (see [9]), so we consider only the case $n_{1} \geq 1$.

We first construct a minimally rigid graph. Consider the case where $n_{1} \geq 2$. Start from one cycle with the vertices of type 1 and one cycle with the vertices of type 2 . If there are only two vertices of a given type, then the cycle is simply an edge between these two vertices and if there is only one vertex of a given type, the cycle is empty. Hence if $n_{i}<3$ for $i \in\{1,2\}$, the corresponding cycle contains $n_{i}-1$ edges and if $n_{i} \geq 3$, the corresponding cycle contains $n_{i}$ edges. In particular, since we assumed that $n_{1} \geq 2$, the cycle with vertices of type 1 has $n_{1}+\min \left(n_{1}-3,0\right)$ edges. If $n_{2}=0$, we are done. If $n_{2} \geq 1$, we select one vertex of type 1 (denoted by $u$ ) and add an edge between this vertex and each vertex of type 2 to get a minimally 
rigid graph. Then for $n_{2} \geq 3$ we are done as the graph has $2 n_{2}+n_{1}+\min \left(0, n_{1}-3\right)$ edges and is sparse. For $n_{2}=1,2$, we need to add an edge, and for example we can add one edge between a vertex of type 1 different from $u$ and any vertex of type 2 .

Consider now the case $n_{1}=1$. The cases $n_{2}=1,2$ are easy, just take the complete graph. For $n_{2} \geq 3$, start as above with a cycle with vertices of type 2 and then add an edge between all vertices of type 2 except one and the vertex of type 1.

We now prove the basis exchange axiom. Let $B_{i}=\left(V, E_{i}\right), i=1,2$ be two minimally rigid graphs and $e_{2} \in E_{2} \backslash E_{1}$. We must show that there exists an edge $e_{1} \in E_{1} \backslash E_{2}$ such that $\left(V, E_{1} \backslash\left\{e_{1}\right\} \cup\left\{e_{2}\right\}\right)$ is minimally rigid. Let $e_{2}=u v$. Consider all the rigid blocks of $B_{1}$ containing vertices $u$ and $v$. By Lemma 3.1, the intersection of these blocks denoted by $B^{\prime}=\left(V^{\prime}, E^{\prime}\right)$ is still a rigid block of $B_{1}$. $B^{\prime}$ is not a rigid block of $B_{2}$, since otherwise the subgraph $E^{\prime} \cup\left\{e_{2}\right\} \subset E_{2}$ would violate the sparsity of $B_{2}$ (note that $u, v \in V^{\prime}$ ). Hence there exists $e_{1} \in E^{\prime} \backslash E_{2}$. We are done if we prove that $B_{3}=\left(V, E_{1} \backslash\left\{e_{1}\right\} \cup\left\{e_{2}\right\}\right)$ is sparse. Consider any subgraph $H$ of $B_{1}$ such that sparsity is violated in $B_{1} \cup\left\{e_{2}\right\}$. Note that $H$ is a rigid block of $B_{1}$ containing both $u$ and $v$. Since $B^{\prime}$ is the minimal subgraph of $B_{1}$ with this property, $B^{\prime} \subseteq H$, and then both endpoints corresponding to $e_{1}$ are in $H$. The addition of $e_{2}$ violates sparsity, but the removal of $e_{1}$ restores the count, and we are done.

Remark 3.4. (due to L. Theran) As pointed out in Remark 2.2. Proposition 3.3 can also be deduced from the fact that, using the terminology of [18, all $(2,0,3)$-gradedsparse graphs form a matroid whose ground set is the set of edges of the complete graph together with two loops at each vertex (see [18]). More precisely, let $M_{1}$ be this matroid with ground set $E_{1}:=\left\{E\left(K_{n}\right) \cup 2\right.$ loops per vertex $\}$ with independent sets $I_{1}$, and let $n_{1}$ and $n_{2}$ be the number of vertices of type 1 (type 2, resp.). Let $L$ be the set of edges containing exactly 1 self-loop at each of the $n_{1}$ vertices of type 1. Consider then $E_{2}:=\left\{E\left(K_{n}\right) \cup L\right\}$ and note that $E_{2} \subseteq E_{1}$. Moreover, $I_{2}:=\left\{A \in I_{1}: A \subseteq E_{2}\right\}$ is still a matroid $M_{2}$, since this corresponds to a truncation of $M_{1}$. Finally, consider the sets $I_{3}:=\left\{A \in I_{2}: A \cup L\right.$ is independent in $\left.M_{2}\right\}$. Since this corresponds to a contraction of $M_{2}$, the resulting structure is a matroid, that corresponds exactly to the matroid described in Proposition 3.3 (all elements of $I_{3}$ being sparse graphs with $n$ vertices, of which $n_{1}$ are of type 1 and $n_{2}$ of type 2 ). In order to make the paper more self-contained, we opted, however, for a direct proof here.

Define a decomposition of the edge set of a graph to be a collection of rigid components such that every edge is exactly in one rigid component, and such that isolated vertices form their own rigid components.

Lemma 3.5. Any graph $G$ decomposes uniquely into rigid components. Any two rigid components intersect in at most one vertex.

Proof. First assume $G$ is sparse. Consider an edge $e=u v$. The edge $u v$ itself is a rigid block. The union of all rigid blocks containing both $u$ and $v$ is, by Lemma 3.1 part (i), still rigid, and this is the unique maximal block $e$ belongs to. If initially we had chosen another edge inside this unique maximal block, the result would clearly be the same (if it were larger, we could again apply Lemma 3.1 part (i) and obtain a bigger block containing $e$ ). Thus, the set of edges forms an equivalence relation whose equivalence classes are given by the rigid components the edges belong to. Isolated vertices always belong to their own component, and hence the 
decomposition is unique, proving the first part for sparse graphs. For such graphs, the second part of the lemma follows immediately from Lemma 3.1 part (ii).

Now, suppose that $G$ is not sparse and consider its rigid components: we will show that we can choose one edge $e=u v$, remove it from $G$ to obtain $G^{\prime}=G \backslash u v$, and that the rigid components of $G^{\prime}$ are the same as those of $G$. Then, repeating the procedure until the graph is made sparse, the lemma will be proved.

First, since rigidity is monotone, a rigid component of $G^{\prime}$ is a rigid block in $G$, and we have to show only that a rigid component in $G$ remains a rigid component in $G^{\prime}$. Since $G$ is not sparse, there exists one subgraph $H$ with $n_{1}(H)+n_{2}(H)$ vertices of type 1 (type 2 , resp.) having more than $n_{1}(H)+2 n_{2}(H)-\min \left\{0, n_{1}(H)-3\right\}$ edges. Among all such subgraphs choose a minimal subgraph $H$, i.e., any induced subgraph of $H$ when leaving out at least one vertex is sparse. Note that $H$ is rigid. Choose the edge $e=u v \in E(H)$. $H \backslash u v$ remains rigid. Let $C$ be a rigid component in $G$. We have to show it is a rigid component in $G^{\prime}=G \backslash u v$. We consider now three cases:

i) $C$ does not contain both $u$ and $v$ : then it remains rigid after the removal of $u v$, and there is nothing else to prove.

ii) $C$ contains $u$ and $v$, but not all of $H$. Then in $G^{\prime}, \tilde{H}:=C \cap H$ is sparse (as every proper subgraph of $H$, and therefore of $H \backslash u v$, is sparse). By the augmentative property of matroids, if not yet spanning, $\tilde{H}$ can be completed to obtain a minimally rigid spanning subgraph $C^{\prime}$ of $C$. Thus $C$ remains rigid in $G^{\prime}$.

iii) $C$ contains all of $H$. Since $H \backslash u v$ is rigid, we can find a minimally rigid spanning subgraph $\tilde{H}$ of $H \backslash u v$. Again, by the augmentative property of matroids, if not yet spanning, $\tilde{H}$ can be completed to obtain a minimally spanning subgraph $C^{\prime}$ of $C$, and $C$ remains rigid in $G^{\prime}$.

Lemma 3.6. Take two rigid components $R_{1}$ and $R_{2}$. Adding at most three pairwise disjoint edges $u_{i} v_{i}$, with $u_{i} \in V\left(R_{1}\right) \backslash V\left(R_{2}\right)$, and $v_{i} \in V\left(R_{2}\right) \backslash V\left(R_{1}\right)$ turns $R_{1} \cup R_{2}$ into a rigid block.

Proof. From the proof of the previous lemma, it suffices to prove the statement for sparse $G$. Remember that two rigid components $R_{1}$ and $R_{2}$ intersect in either 0 or 1 vertex, and by monotonicity we may assume that there is no edge from $V\left(R_{2}\right) \backslash V\left(R_{1}\right)$ to $V\left(R_{1}\right) \backslash V\left(R_{2}\right)$. Let $n_{1}\left(R_{1}\right)=i$ and $n_{1}\left(R_{2}\right)=j$. If $R_{1} \cap R_{2}=\emptyset$, then let $t=0$, and otherwise let $t \in\{1,2\}$ be equal to the type of the vertex in $R_{1} \cap R_{2}$. If $t \in\{1,2\}$ and $\min \{i, j\} \geq 3$, by Lemma 3.1 part(ii), $R_{1} \cup R_{2}$ is already rigid. By a similar argument as in the proof of Lemma 3.1 part(ii), we can show that the case $i \geq 3, j=2$ and $t=2$ is impossible, as in this case $m\left(R_{1} \cap R_{2}\right) \geq 1$, and thus $n\left(R_{1} \cap R_{2}\right) \geq 2$. In all other cases, do the following: if $\min \{i, j\} \geq 3$ and $t=0$, then no edge is added. If $i \geq 3$ and $j<3,3-j-t$ edges are added, if $i<3$ and $j<3$ and $(i+j) \geq 3$, then $6-i-j-t$ edges are added, and if $i<3$ and $j<3$ and $(i+j)<3$, then $3-t$ edges are added. It can be seen that in all cases the total number of edges needed for $R_{1} \cup R_{2}$ being minimally rigid is correct. Moreover, the number of edges added is for any fixed value of $i$ monotone nondecreasing in $j$. Also, for non-intersecting subgraphs $A \subseteq R_{1}$ and $B \subseteq R_{2}$ the number of edges that can be added between $A$ and $B$ without violating sparsity is at least the number that can be added in case they intersect in one vertex. In particular, this means that for any subgraph $A \subseteq R_{1}$ with $i^{\prime} \leq i$ vertices of type 1 and any subgraph $B \subseteq R_{2}$ with $j^{\prime} \leq j$ vertices of type 1 such that $\min \{n(A), n(B)\} \geq 2$, the number 
of edges that can be added between vertices of $A$ and $B$ without violating sparsity is at least the number of edges added between $R_{1}$ and $R_{2}$, and thus such subgraphs remain sparse. Otherwise, suppose $n(A)=1$ and we may assume $A$ and $B$ disjoint. By disjointness of the newly added edges at most 1 edge is added between $A$ and $B$. Thus, an originally sparse graph $B$ remains sparse after adding one vertex and at most one edge. Thus, all subgraphs are sparse, and the statement follows.

\section{Proof of Theorem 2.14}

The proof of Theorem 2.14 relies on Theorem 3 in 11 and Lemma 2.22 .

To a simple graph $G=(V, E)$, we associate the bipartite graph $G^{b}=\left(V^{b}, E^{b}\right)$ with vertex set $V^{b}=V \cup E$ and an edge between $v \in V$ and $e \in E$ if and only if $v$ is an end-point of $e$ in $G$. We say that $G^{B}$ is the bipartite version of $G$. The size of a spanning subgraph $S=(V \cup E, F)$ of $G^{b}$ is defined as the number of edges $|F|$ of $S$. We now consider the case where each vertex of the original graph $V$ has a type in $\{1,2\}$. We say that a spanning subgraph is admissible if for each $v \in V$, the degree of $v$ in $S$ is at most its type and for each $e \in E$, the degree of $e$ in $S$ is at most one.

Clearly, if $G$ is 1.5-orientable, an orientation gives a spanning subgraph with size $|E|$ which is the maximum possible size of an admissible spanning subgraph. Hence we have the following claim: a graph $G$ is 1.5-orientable if and only if the size of a maximum admissible spanning subgraph of $G^{b}$ is equal to $|E|$.

For the random graph $G(n, c / n)$, when types are drawn independently at random being 1 with probability $1-q$, and 2 otherwise, independently of the rest, we denote by $M_{n}=M_{n}(c, q)$ the size of the largest admissible spanning subgraph of the bipartite version $G_{n}^{b}$ of $G(n, c / n)$. Our previous claim translates into: $G(n, c / n)=$ $\left(V_{n}, E_{n}\right)$ is 1.5-orientable if and only if $M_{n}=\left|E_{n}\right|$.

The fact that $G_{n}^{b}$ satisfies the assumption of Theorem 3 [11] is proved in Section 6 of [11. With the notation of Theorem 3 in [11, we choose the set $A_{n}$ equal to $E_{n}$ and the set $B_{n}$ equal to $V_{n}$, so that we have $D^{A}=2, W^{A}=1$ and

$$
D^{B}=\operatorname{Poi}(c), \quad \text { and } \quad W^{B}= \begin{cases}1 & , \text { w.p. } 1-q \\ 2 & , \text { w.p. } q .\end{cases}
$$

We now compute $\inf _{x \in[0,1]} \mathcal{F}^{A}(x)$, which is according to Theorem 3 in [11] the value for the $\operatorname{limit}_{n \rightarrow \infty} \lim _{n \rightarrow \infty} \frac{M_{n}}{\left|E_{n}\right|}$. Using the definitions given in Theorem 3 of [11], we have

$$
\begin{aligned}
g^{A}(x) & =1-x \\
g^{B}(x) & =1-(1-q) Q(c x, 1)-q Q(c x, 2) \\
\mathcal{F}^{A}(x) & =1-\left(1-g^{B}(x)\right)^{2}+\frac{2}{c}((1-q) Q(c x, 2)+2 q Q(c x, 3)) .
\end{aligned}
$$

From Theorem 3 in [11, we know that $\mathcal{F}^{A}(x)$ is minimized only for $x$ solving $x=g^{A} \circ g^{B}(x)$. More precisely, the derivative of $\mathcal{F}^{A}(x)$ has the same sign as $\Delta(x)=x-g^{A} \circ g^{B}(x)$. Then, we have (note that $\frac{d}{d x} Q(x, y)=e^{-x} \frac{x^{y-1}}{(y-1) !}$ )

$$
\begin{aligned}
\Delta(x) & =x-(1-q) Q(c x, 1)-q Q(c x, 2) \\
\Delta^{\prime}(x) & =1-c(1-q) e^{-c x}-c^{2} x q e^{-c x} \\
\Delta^{\prime \prime}(x) & =c^{3} q e^{-c x}\left(x-\frac{2 q-1}{c q}\right)
\end{aligned}
$$


Note that $\Delta(0)=0, \Delta(1)>0$. Define $\tilde{x}=\tilde{x}(c, q)$ as the largest solution in $[0,1]$ to the equation $\Delta(x)=0$. Note that $\mathcal{F}^{A}(0)=1$. Moreover we have

$$
\Delta^{\prime}(1)=1-c(1-q) e^{-c}-c^{2} q e^{-c} \geq 1-c e^{-c} \geq 1-e^{-1} \geq 0 .
$$

We now prove that

$$
\inf _{x \in[0,1]} \mathcal{F}^{A}(x)=\min \left\{1, \mathcal{F}^{A}(\tilde{x})\right\} .
$$

First assume that $q \leq 1 / 2$ so that, we have $\Delta^{\prime \prime}(x) \geq 0$ for $x \in[0,1]$. If $1-q \leq 1 / c$, then $\Delta^{\prime}(0)=1-c(1-q) \geq 0$ and we have $\Delta(x) \geq 0$ so that $\tilde{x}=0$ and the function $\mathcal{F}^{A}(x)$ is increasing. Hence $\inf _{x \in[0,1]} \mathcal{F}^{A}(x)=\mathcal{F}^{A}(0)=1$. Now if $1-q>1 / c$, then a similar analysis shows that $\mathcal{F}^{A}(x)$ is decreasing on $[0, \tilde{x}]$ and increasing on $[\tilde{x}, 1]$, so that we have $\inf _{x \in[0,1]} \mathcal{F}^{A}(x)=\mathcal{F}^{A}(\tilde{x})<1$.

Assume now that $q>1 / 2$ so that $\Delta^{\prime \prime}(x)$ vanishes once on $(0,1)$. Hence, if $1-q>1 / c$, we have $\Delta^{\prime}(0)<0$ so that $\Delta(x)<0$ for $x \in(0, \tilde{x})$ and $\Delta(x)>0$ for $x \in(\tilde{x}, 1]$. As above, we have $\inf _{x \in[0,1]} \mathcal{F}^{A}(x)=\mathcal{F}^{A}(\tilde{x})<1$. Consider then the case $1-q \leq 1 / c$, so that $\Delta^{\prime}(0) \geq 0$. Moreover as $\Delta^{\prime}(1) \geq 0$, either $\Delta(x)$ is non-negative or there exists $0<y<\tilde{x}$ such that $\Delta(x)$ is positive on $(0, y)$ and $(\tilde{x}, 1)$ and negative on $(y, \tilde{x})$. In any case, we have $\inf _{x \in[0,1]} \mathcal{F}^{A}(x)=\min \left\{\mathcal{F}^{A}(0), \mathcal{F}^{A}(\tilde{x})\right\}$, and $\sqrt{6}$ is proved.

By Theorem 3 in [11, we have

$$
\lim _{n \rightarrow \infty} \frac{M_{n}}{\left|E_{n}\right|}=\inf _{x \in[0,1]} \mathcal{F}^{A}(x)=\min \left\{1, \mathcal{F}^{A}(\tilde{x})\right\} .
$$

In the argument above, we showed that for $1-q>1 / c$, we have $\tilde{x}>0$ and $\mathcal{F}^{A}(\tilde{x})<1$ and for $1 / 2 \leq 1-q \leq 1 / c$, we have $\tilde{x}=0$ and $\inf _{x \in[0,1]} \mathcal{F}^{A}(x)=1$. In particular, we see that for $1-q>1 / c, G(n, c / n)$ is not 1.5-orientable and for $1 / 2 \leq 1-q \leq 1 / c, G(n, c / n)$ is 'almost' 1.5-orientable, i.e., all vertices except possibly $o(n)$ will satisfy their indegree constraints. We will show that the graph is indeed 1.5-orientable in the last part of the proof relying on Lemma 2.22.

Before that, we consider the case $q>1 / 2$ and find the condition on $c$ for $\mathcal{F}^{A}(\tilde{x})<$ 1 , where $\tilde{x}=\tilde{x}(c)$ is the largest solution to $\Delta(x)=0$ with $\Delta(x)$ defined in (5). First note that by the previous analysis, if $\mathcal{F}^{A}(\tilde{x})<1$ and $\Delta(\tilde{x})=0$, then $\tilde{x}$ is necessarily the largest solution to $\Delta(x)=0$.

Now using the fact that $\Delta(\tilde{x})=0$, we see that $g^{B}(\tilde{x})=1-\tilde{x}$, so that we have

$$
\mathcal{F}^{A}(\tilde{x})=1-\tilde{x}^{2}+\frac{2}{c}((1-q) Q(c \tilde{x}, 2)+2 q Q(c \tilde{x}, 3)) .
$$

Making the change of variable $\xi=c \tilde{x}$, we get

$$
\mathcal{F}^{A}(\tilde{x})=1-\frac{\xi^{2}}{c^{2}}+\frac{2}{c}((1-q) Q(\xi, 2)+2 q Q(\xi, 3)) .
$$

Define the function

$$
f(\xi, q)=\xi \frac{(1-q) Q(\xi, 1)+q Q(\xi, 2)}{(1-q) Q(\xi, 2)+2 q Q(\xi, 3)}
$$

and recall that the definition of $\tilde{x}$ becomes for $\xi$ :

$$
\xi=c((1-q) Q(\xi, 1)+q Q(\xi, 2)),
$$

so that we can rewrite the previous expression as

$$
\mathcal{F}^{A}(\tilde{x})=1-\frac{1}{c}(f(\xi, q)-2)((1-q) Q(\xi, 2)+2 q Q(\xi, 3)) .
$$


We have $\mathcal{F}^{A}(\tilde{x})<1$ if and only if $f(c \tilde{x}, q)>2$. Note that for $q>0$, we have $\lim _{\xi \rightarrow 0} f(\xi, q)=2$ and $\lim _{\xi \rightarrow 0} f(\xi, 0)=3 / 2$. Moreover, for any $q>1 / 2$, one can show that there exists a unique positive solution to $f\left(\xi^{*}, q\right)=2$ and for $\xi \in\left(0, \xi^{*}\right)$, we have $f(\xi, q)<2$, while for $\xi>\xi^{*}$, we have $f(\xi, q)>2$. Now, using (7), we define

$$
c^{*}=\frac{\xi^{*}}{(1-q) Q\left(\xi^{*}, 1\right)+q Q\left(\xi^{*}, 2\right)},
$$

so that $c^{*} \tilde{x}\left(c^{*}\right)=\xi^{*}$.

Note that as a function of $c$ with $x$ fixed, $\Delta(x)$ is non-increasing in $c$ which implies that $\tilde{x}(c)$ is non-decreasing in $c$ (note that for $q>1 / 2$, by the previous analysis, $\Delta(x)$ is always negative on the left of $\tilde{x}$ and positive on its right).

Thanks to the monotonicity of $c \mapsto \tilde{x}(c)$, we have: if $c>c^{*}$, then $c \tilde{x}(c)>\xi^{*}$ and $f(c \tilde{x}, q)>2$, i.e., $\mathcal{F}^{A}(\tilde{x})<1$. Similarly, if $c<c^{*}$ and $\tilde{x}(c)>0$, then we get $f(c \tilde{x}, q)<2$, i.e., $\mathcal{F}^{A}(\tilde{x})>1$.

To summarize, we proved that for the function $c^{*}(q)$ defined in the statement of the theorem, we have: for $c<c^{*}(q)$, a.a.s., we have $\lim _{n \rightarrow \infty} \frac{M_{n}}{\left|E_{n}\right|}=1$ and for $c>c^{*}(q)$, we have $\lim _{n \rightarrow \infty} \frac{M_{n}}{\left|E_{n}\right|}<1$. In particular, (b) follows: if $c>c^{*}(q)$, the graph is a.a.s. not 1.5-orientable. We still need to prove that if $c<c^{*}(q)$, the graph is a.a.s. 1.5-orientable.

Choose $\tilde{c}$, such that $c<\tilde{c}<c^{*}$. Let $\tilde{G}_{n}$ be an associated random graph and $\tilde{M}_{n}$ a maximum admissible subgraph. Construct a coupling between random graphs with different parameters $c<\tilde{c}$, by removing edges in $\tilde{G}_{n}$ with the appropriate probability. The goal is to construct an admissible subgraph $\bar{M}_{n}$ for some $G_{n}(\bar{c})$ with $\bar{c} \geq c$ such that $\left|\bar{M}_{n}\right|=\left|\bar{A}_{n}\right|$. In other words, $G_{n}(\bar{c})$ is 1.5-orientable which implies the claim as $G_{n}(n, c / n)$ can be obtained from $G_{n}(\bar{c})$ by removing edges.

If $\left|\tilde{M}_{n}\right|=\left|\tilde{A}_{n}\right|$, we are done. Assume then that $\left|\tilde{M}_{n}\right|<\left|\tilde{A}_{n}\right|$ and consider the bipartite graph $\tilde{G}_{n}^{b}$. We say that a vertex $v \in \tilde{V}_{n}$ is saturated if its degree in $\tilde{M}_{n}$ is equal to its type. Note that if an edge $(v, e) \in E\left(\tilde{G}_{n}^{b}\right)$ where $v \in \tilde{V}_{n}$ and $e \in \tilde{E}_{n}$ is not covered by $\tilde{M}_{n}$, then the vertex $v$ is saturated (otherwise $\tilde{M}_{n}$ would not be maximal hence not maximum). In particular, if $e \in \tilde{E}_{n}$ is isolated in $\tilde{M}_{n}$, then each of its neighbors $u$ and $v$ is saturated. Starting from these vertices and following the covered edges, we can then construct alternating paths in which the edges are alternatively covered in $\tilde{M}_{n}$ and uncovered. Let $\tilde{K}^{b}$ be the union of all such alternating paths (see Figure 1 for an illustration).

Each vertex $v \in \tilde{V}_{n} \cap \tilde{K}^{b}$ is saturated so that the graph $\tilde{K}$ associated to $\tilde{K}^{b}$ in the original $\tilde{G}_{n}$ is a subgraph satisfying the hypotheses of Lemma 2.22 . Hence there exists $\alpha>0$ such that $\tilde{K}$ has size (i.e., number of vertices $|V(\tilde{K})|$ ) at least $\alpha$.

Let us call $\mathrm{g} a p_{n}=\left|\tilde{E}_{n}\right|-\left|\tilde{M}_{n}\right|$. By the previous analysis, we know that gap $p_{n}=$ $o(n)$. To make the coupling between random graphs at different $c$ 's explicit, attach a uniform random variable $\mathcal{U}_{[0,1]}$ to each edge of $\tilde{G}_{n}$, rank the edges according to these variables, and delete them sequentially to construct the graphs $G_{n}(c)$ with $c<\tilde{c}$. Since $|V(\tilde{K})| \geq \alpha$, each time an edge is removed, the probability it belongs to $\tilde{K}$ is larger than $\varepsilon^{\prime}$, for some $\varepsilon^{\prime}>0$. Moreover, each time an edge in $\tilde{K}$ is removed, gap $p_{n}$ decreases by one. Thus the probability that deleting an edge decreases gap is at least $\varepsilon^{\prime}$. Hence a.a.s. gap $p_{n}$ reaches 0 before the graph $G_{n}$ with parameter $c$ is constructed. At this point, we have found $\bar{c} \geq c$ such that $\left|\bar{M}_{n}\right|=\left|\bar{A}_{n}\right|$ and we have proved that a.a.s. $G_{n}(n, c / n)$ is 1.5 -orientable: we are done. 


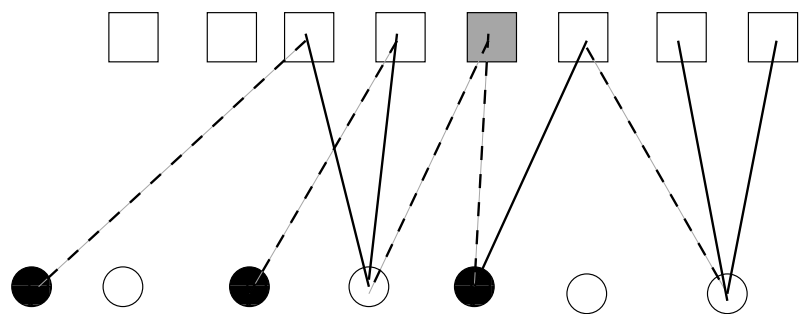

FiguRE 1. Bipartite representation of $G_{n}$ and construction of the alternating paths. Squares (upper row) are the edges of $G_{n}$; circles (lower row) are the vertices of $G_{n}$. Filled (resp. empty) circles are vertices of type 1 (resp. type 2 ). Solid lines (resp. dashed lines) are edges in $\tilde{G}_{n}^{b}$ that are (resp. are not) in $\tilde{M}_{n}$. Note that not all edges of $\tilde{G}_{n}^{b}$ are represented. The grey square is an edge of $G_{n}$ which is not covered by $\tilde{M}_{n}$ : thus it is a starting point for alternating paths.

\section{ORIENTABILITY AND RIGIDITY}

In this section we will show that the threshold of having a giant rigid component coincides with the threshold for 1.5-orientability, which together with Theorem 2.14 completes the proof of Theorem 2.10

One part of the equivalence of the thresholds of rigidity and 1.5-orientability is easy:

Lemma 5.1. If for some $c, G$ contains a rigid component $H$ of linear size, then a.a.s., for all $c^{\prime} \geq c+\epsilon$ and $\epsilon>0, G$ is not 1.5-orientable.

Proof. This lemma is similar to Lemma 5.1 of 7 . If $G$ contains a giant rigid component $H$, then $|V(H)|=n_{1}^{\prime}+2 n_{2}^{\prime} \geq \alpha$ for some $\alpha>0$ and $|E(H)| \geq$ $n_{1}^{\prime}+2 n_{2}^{\prime}-3$. When considering $c^{\prime}=c+\epsilon$ for some $\epsilon>0$, then note that a graph in $G^{\prime} \in \mathcal{G}\left(n, c^{\prime} / n\right)$ can be obtained from $G$ by adding a fresh random graph with parameters $G^{\prime \prime} \in \mathcal{G}(n, \epsilon(1+o(1)) / n)$, where the $o(1)$ part accounts for edges present in the intersection of both graphs. When adding the fresh random graph, $\Theta(n)$ edges will be added with probability $1-e^{-\Omega(n)}$. For each such edge, there is positive probability that both of its endpoints are in $H$, and thus for any fixed $\epsilon>0$, a.a.s., at least 4 edges will be added to $E(H)$, and for $c^{\prime},|E(H)|>n_{1}^{\prime}+2 n_{2}^{\prime}$. Using Proposition 2.13, $G$ is not 1.5-orientable, and the lemma follows.

The other direction is harder. For $q=1$ (all sites are type 2, standard 2D rigidity percolation), the authors of [7] use a lemma by Theran ([19]), stating that rigid components have size at most 3 , or they are of size $\Omega(n)$. This is not true for $q<1$, and we will use Lemma 2.22 instead. We will first make one simple observation.

Lemma 5.2. Let $q<1$ and let $G \in \mathcal{G}(n, c / n)$ with $c>\frac{1}{1-q}$. A.a.s., $G$ contains a giant rigid connected block.

Proof. We will show that the subgraph induced by the vertices of type 1 contains a giant rigid block: indeed, note that the subgraph $G^{\prime} \in \mathcal{G}((1-q) n, c / n)=\mathcal{G}((1-$ $\left.q) n, \frac{c(1-q)}{(1-q) n}\right)$. Since we are interested in the asymptotic behavior of such graphs, 
we may replace $(1-q) n$ by $n$, and the behavior of such a graph is like the one of $G^{\prime} \in \mathcal{G}\left(n, \frac{c(1-q)}{n}\right)=\mathcal{G}\left(n, c^{\prime} / n\right)$ for some $c^{\prime}>1$. By standard results (see for example [2]), a.a.s., $\mathcal{G}\left(n, \frac{c^{\prime}-\left(c^{\prime}-1\right) / 2}{n}\right)$ contains a giant connected component, and by adding a fresh random graph $G^{\prime \prime} \in \mathcal{G}\left(n, \frac{(1+o(1))\left(c^{\prime}-1\right) / 2}{n}\right)$, a.a.s. at least one cycle will be added. Hence, by possibly removing edges, we may pick a connected subgraph $H$ of linear size containing exactly one cycle. $H$ is the desired rigid block, since $|V(H)|=|E(H)|$, and every subgraph of $H$ satisfies the sparsity condition. The statement follows.

We need one more helper lemma.

Lemma 5.3. There is $\alpha>0$ such that: if $G$ is a $G(n, c / n)$ random graph with $c>2$, and $G$ is not Laman sparse, then, a.a.s., $G$ spans a rigid connected block of size at least $\alpha$.

Proof. Assume first that $G$ is a $G\left(n, c_{0} / n\right)$ random graph with $c_{0}>2$, and has only type 2 vertices. If $G$ is not Laman sparse, then by Lemma 4.2 of [7, $G$ spans a rigid component on at least four vertices. Now, by Proposition 3.3 of [7, a.a.s. all rigid components of $G$ have size 1,2,3 or $\Omega(n)$. In fact, by applying Proposition 4 of [19] with $a=2$, we see that a.a.s. such a rigid component is of size at least $\left(\left(4 / c_{0}\right)^{2} e^{-3}\right) n$. Since rigidity is preserved by addition of edges, this statement is also true for any $c \geq c_{0}$. Note that these rigid components with only type 2 vertices are connected. Now, according to Lemma 3.2 a rigid subgraph of $G$ remains rigid if some vertices of $G$ are changed from type 2 to type 1 . Hence, for any $c \geq c_{0}>2$, $G \in \mathcal{G}(n, c / n)$ spans a rigid connected block which is of size at least $\left(\left(4 / c_{0}\right)^{2} e^{-3}\right) n$. The statement of the lemma follows with $\alpha=4 e^{-3}$, taking the limit as $c_{0} \rightarrow 2$.

We are now able to prove the counterpart to Lemma 5.1 .

Lemma 5.4. Suppose that $G \in \mathcal{G}(n, c / n)$ is not 1.5-orientable. Then, for any $\epsilon>0$, a.a.s., $G \in \mathcal{G}(n,(c+\varepsilon) / n)$ contains a giant rigid connected block $H$.

Proof. Assume now that $G$ is not 1.5-orientable. We have shown in Theorem 2.14 that the threshold for 1.5 -orientability $c^{*}(q)$ satisfies $c^{*}(q)=\frac{1}{1-q}$ for $q \in[0,1 / 2]$ and $c^{*}(q)<\frac{1}{1-q}$ for $q \in(1 / 2,1]$, and since we are interested in a statement that holds a.a.s., we may assume $c \geq c^{*}(q)$. For $q \in[0,1 / 2]$, for any $\varepsilon>0, c+\varepsilon>\frac{1}{1-q}$, and by Lemma 5.2 , a.a.s. $G$ contains a connected giant rigid component.

We may therefore assume $q>1 / 2$ and $c<\frac{1}{1-q}$ and have to show that in this case a.a.s. $G \in \mathcal{G}(n, c / n)$ contains a giant rigid connected block $H$, implying the statement since the property of containing a giant rigid connected block is monotone. Notice that for $q>1 / 2, c^{*}(q)>2$; since we may assume $c \geq c^{*}(q)$, we may assume $c>2$. By Proposition 2.13 there exists a subgraph $H \subseteq G$ with $n_{1}^{\prime}$ vertices of type $1, n_{2}^{\prime}$ vertices of type 2 , such that $|E(H)|=m^{\prime}>n_{1}^{\prime}+2 n_{2}^{\prime}$. Among all such subgraphs, let $H$ be minimal with respect to the number of vertices for this property (if there are several choices, pick an arbitrary such $H$ ). By minimality, $H$ is connected. Since now $c<\frac{1}{1-q}$, by Lemma 2.22 a.a.s., $|V(H)| \geq \alpha n$ for some $\alpha=\alpha\left(q, c-\frac{1}{1-q}\right)$. Now we have to construct a giant rigid connected block starting from $H$. We will now show that either $G$ contains a connected giant rigid block or any arbitrary subgraph $\tilde{H} \subseteq H$ with $V(\tilde{H}) \neq V(H)$ fulfills the sparsity condition. 
Consider an arbitrary subgraph $\tilde{H} \subseteq H$ with $V(\tilde{H}) \neq V(H)$, and let $\tilde{n}_{1}$ and $\tilde{n}_{2}$ be the numbers of type 1 and type 2 vertices of $\tilde{H}$, respectively. By minimality of $H$,

$$
|E(\tilde{H})| \leq \tilde{n}_{1}+2 \tilde{n}_{2} .
$$

Consider first the case $\tilde{n}_{1}=0$. If $|E(\tilde{H})| \geq 2 \tilde{n}_{2}-2, G$ is not Laman sparse, $c>2$, hence Lemma 5.3 ensures that $G$ contains a giant rigid connected block, and we are done. Otherwise, $|E(\tilde{H})| \leq 2 \tilde{n}_{2}-3$ for any subgraph $\tilde{H}$ with $\tilde{n}_{1}=0$, and these subgraphs are thus sparse. In this case we consider then subgraphs with $\tilde{n}_{1}=1$. If $|E(\tilde{H})|>2 \tilde{n}_{2}-1$, then $|E(\tilde{H})|>2\left(\tilde{n}_{1}+\tilde{n}_{2}\right)-3$, and again $G$ is not Laman sparse, and as before Lemma 5.3 ensures that $G$ contains a giant rigid connected block, and we are done. Otherwise, $|E(\tilde{H})| \leq 2 \tilde{n}_{2}-1$ for all subgraphs with $\tilde{n}_{1}=1$ holds, and these subgraphs are sparse as well. In this case we consider the case $\tilde{n}_{1}=2$. If $|E(\tilde{H})|>2 \tilde{n}_{2}+1$, then $|E(\tilde{H})|>2\left(\tilde{n}_{1}+\tilde{n}_{2}\right)-1$, and by the same argument as before, by Lemma 5.3, $G$ contains a giant rigid connected block, and we are done. Otherwise, for all subgraphs with $\tilde{n}_{1}=2$, we have $|E(\tilde{H})| \leq 2 \tilde{n}_{2}+1$, and these subgraphs are sparse as well. We then consider subgraphs $\tilde{n}_{1} \geq 3$. For them, $\tilde{H}$ clearly verifies the sparsity condition. Thus, either we have found a giant rigid connected block in $G$, or all proper subgraphs of $H$ fulfill the sparsity condition. In the latter case, since $|E(H)|>n_{1}^{\prime}+2 n_{2}^{\prime}$, we can remove some edges from $H$ so that $|E(H)|=n_{1}^{\prime}+2 n_{2}^{\prime}$ (in the case $n_{1}^{\prime} \geq 3$ ), or $|E(H)|=n_{1}^{\prime}+2 n_{2}^{\prime}-1$ (in the case $n_{1}^{\prime}=2$ ), or $|E(H)|=n_{1}^{\prime}+2 n_{2}^{\prime}-2$ (in the case $n_{1}^{\prime}=1$ ), or $|E(H)|=2 n_{2}^{\prime}-3$ (in the case $n_{1}^{\prime}=0$ ), and in all cases, since $|E(H)| \geq|V(H)|$, this can be done without disconnecting $H$. In this way $H$ is minimally rigid, connected, and since $|V(H)| \geq \alpha n$ for some $\alpha=\alpha\left(q, c-\frac{1}{1-q}\right), H$ provides the giant rigid connected block.

Combining Lemma 5.1 and Lemma 5.4, we see that the thresholds for 1.5orientability and rigidity coincide, and together with Theorem 2.14 the proof of the first part of Theorem 2.10 is completed.

We turn now to the uniqueness statement of Theorem 2.10 adapting the proof of [7. Note first that since the size of each giant rigid connected block is at least $n / \omega_{n}$ for some function $\omega_{n}$ that arbitrarily slowly tends to $\infty$ as $n \rightarrow \infty$, and since by Lemma 3.1(i) any two rigid blocks intersect in at most one vertex, there can be at most $\omega_{n}(1+o(1))$ such blocks (we suppose connected blocks here to be giant inclusion-wise maximal connected blocks).

We generate $G \in \mathcal{G}(n, c / n)$ as follows. Start with the empty graph; order randomly the edges in $K_{n}$; then add sequentially the first $m$ edges according to this ordering, with $m \sim \operatorname{Bin}\left(\left(\begin{array}{l}n \\ 2\end{array}\right), c / n\right)$. At time $t$, the graph under construction thus has $t$ edges.

Define $s:=\omega_{n}^{3} \log n$ and suppose now that at some time $t_{0}$ we have two connected rigid blocks $R_{1}$ and $R_{2}$, each of size at least $n / \omega_{n}$ for some function $\omega_{n}$ tending to infinity arbitrarily slowly as $n \rightarrow \infty$, such that $R_{1} \cup R_{2}$ is not yet a connected rigid block. Lemma 3.6 ensures that is enough to add 3 pairwise disjoint edges between $R_{1}$ and $R_{2}$ to make $R_{1} \cup R_{2}$ a giant connected rigid block. The probability that $R_{1} \cup R_{2}$ is not a connected rigid block by time $t_{0}+s$ is at most

$$
\left(1-\Omega\left(\left(\frac{1}{\omega_{n}}\right)\right)^{2}\right)^{s}=O\left(n^{-\omega_{n}}\right) .
$$


Since there are at most $r:=\omega_{n}(1+o(1))$ (giant inclusion-wise maximal) connected rigid blocks, after $r-1$ mergings the union of all such blocks forms a unique connected rigid block: this can be seen by considering an auxiliary graph whose vertices are giant connected rigid blocks, and an edge between two vertices is added if the union of the blocks is a connected rigid block; once this auxiliary graph is a tree, the union of all blocks is also a connected rigid block. Therefore, with probability $1-O\left(\omega_{n} n^{-\omega_{n}}\right)=1-O\left(n^{-\omega_{n}}\right)$ there are in total at most $\omega_{n}(1+o(1)) s$ steps with more than one giant connected rigid block.

The probability that $m$ equals any fixed number of edges is $O(1 / \sqrt{n})$, and hence the probability that $m$ equals one fixed number having one more than one giant connected rigid block is at most $O\left(s \omega_{n} / \sqrt{n}\right)=o(1)$. Since after exactly $m$ steps we obtain $G \in \mathcal{G}(n, c / n)$, a.a.s. there is only one unique rigid giant connected block in $G$, and thus a.a.s. clearly also one unique giant rigid component, and Theorem 2.10 follows.

\section{Proof of Theorem 2.11}

We first prove the following auxiliary lemmas.

Lemma 6.1. Let $G$ be a rigid graph. Then for $i \in\{1,2\}$, any vertex of type $i$ has degree at least $i$ in $G$.

Proof. Let $\bar{G}$ be a spanning minimally rigid subgraph of $G$, and suppose that $v$ is a vertex of type $i$ with degree strictly less than $i$ in $\bar{G}$. Considering the cases $n_{1}^{\prime}=0,1,2$ and $n_{1}^{\prime} \geq 3$ separately, we see that in each case $\bar{G} \backslash\{v\}$ is a subgraph of $\bar{G}$ which violates the sparsity condition, contradicting the sparsity of $\bar{G}$.

Lemma 6.2. Let $G$ be a rigid graph, and $v$ a type $i$ vertex with degree $i$. Then $G \backslash\{v\}$ is rigid (but not necessarily connected).

Proof. Let $\bar{G}$ be a spanning, minimally rigid subgraph of $G$. The degree of $v$ in $\bar{G}$ is by Lemma 6.1 still $i$.

First, $\bar{G} \backslash\{v\}$ is sparse, since $\bar{G}$ is sparse. If $v$ is of type 2, removing $v$ removes two degrees of freedom and two edges, so the constraint of the total number of edges counting for minimal rigidity is satisfied for $\bar{G} \backslash\{v\}$. Note, however, that $\bar{G} \backslash\{v\}$ might be the disjoint union of two rigid blocks with no edge in between (in which case, by sparsity, both blocks $H_{1}$ and $H_{2}$ satisfy $n_{1}\left(H_{i}\right) \geq 3$ ). If $v$ is type 1 and if also $n_{1}(G)>3$, then removing $v$ removes one degree of freedom and one edge, so the constraint counting for minimal rigidity is also satisfied for $\bar{G} \backslash\{v\}$.

It remains to consider the case $v$ of type 1 and $n_{1}(G) \leq 3$. If we had $n_{1}(G)=1$, then $\bar{G} \backslash\{v\}$ is sparse with $n_{1}(\bar{G} \backslash\{v\})=0$, and we would have

$$
m(\bar{G} \backslash\{v\}) \leq 2 n(\bar{G} \backslash\{v\})-3 .
$$

This would imply for $\bar{G}$

$$
m(\bar{G})=m(\bar{G} \backslash\{v\})+1 \leq 2 n_{2}(\bar{G})-2=n_{1}(\bar{G})+2 n_{2}(\bar{G})-3,
$$

contradicting the rigidity of $\bar{G}$. The same reasoning excludes the cases $n_{1}(G)=2$ and $n_{1}(G)=3$, and the proof follows.

Lemma 6.3. Let $C$ be a rigid block of a graph $G, K_{2.5}$ the 2.5-core of $G$, and $K_{2.5}^{+}$ its $2.5+1.5$-core. If the 2.5 -core of $C$ is not empty, then $C \subseteq K_{2.5}^{+}$. 
Proof. Let us recursively remove vertices to construct the 2.5-core of $C . C_{0}:=C$ is rigid, thus by Lemma 6.1, the first removed vertex $v_{1}$, assumed to be of type $i$, has degree $i$. By Lemma 6.2. $C_{1}=C \backslash\left\{v_{1}\right\}$ is rigid. We can iterate this process until the 2.5-core of $C$ is constructed. At each step we can apply Lemmas 6.1 and 6.2 , hence we only remove type $i$ vertices with degree exactly $i$. The union of all remaining vertices remaining form the 2.5-core of $C$. Since the 2.5-core of $C$ is not empty and is a subgraph of $K_{2.5}$, all the removed vertices are in the $2.5+1.5$-core. Therefore $C \subseteq K_{2.5}^{+}$.

Collecting all previous results, it is now not hard to prove Theorem 2.11 .

Consider first the case $q>1 / 2$. Since the threshold for the existence of a giant rigid component $c^{*}(q)$ satisfies $c^{*}(q)<\frac{1}{1-q}$, by monotonicity of the property of having a giant rigid component, it suffices to show the claim for $c<\frac{1}{1-q}$. By looking at the proof of Lemma 5.4 , either $G$ is not Laman-sparse, and then by Lemma 5.2 , there exists some $\alpha>0$ such that the size of the giant rigid component is at least $\alpha n$, or a rigid giant component $H$ is found, which by Lemma 2.22 is also of size at least $\alpha n$ for some $\alpha>0$. Thus, the transition is discontinuous for all $q>1 / 2$, and the first part of the statement of Theorem 2.11 follows.

Consider now the case $q \leq 1 / 2$. Denote by $H$ the largest rigid component. Consider $c=\frac{1}{1-q}+\varepsilon$ for any fixed $\varepsilon>0$. Take $c=\frac{1}{1-q}+\varepsilon, \varepsilon>0$. By Lemma 5.2. for $c=\frac{1}{1-q}+\varepsilon / 2$, the subgraph of the vertices of type 1 contains a giant component. Moreover, by a result of 1 , this subgraph contains a.a.s. a path of length at least $(\varepsilon / 2)^{2}(1-q) n / 5$. By adding a fresh random graph $G^{\prime \prime} \in \mathcal{G}\left(n, \frac{(1+o(1))(\varepsilon / 2)}{n}\right)$ (as before, the $o(1)$ term is for the intersection, so that we end up with $G \in \mathcal{G}(n, c / n)$ ), a.a.s. among these vertices a cycle of length $\mu \varepsilon^{2} n$ for some small $\mu>0$ is created. Since this is a cycle containing vertices of type 1 only, this cycle is a rigid block, and hence $R_{n}(q, c) / n$ is at least $\mu \varepsilon^{2}$. Imagine now that this cycle of type 1 vertices is not included in $H$ : if one of the vertices of the cycle would be included in $H$ and $n_{1}(H) \geq 3$, then we could add all other vertices of the cycle in a path like way, that is, for each vertex of the cycle not yet present add the vertex together with exactly one incident edge, and the graph remains minimally rigid (in particular, if for a vertex both of its neighbors on the cycle are already there, add just one edge; in particular, the cycle of type 1 is contained in $H$ ). Similarly, if one vertex of the cycle would be included in $H$ and $n_{1}(H)=2$, we could add all vertices of the cycle, including the edge closing the cycle (so that the total number of edges is right). If one vertex of the cycle would be included in $H$ and $n_{1}(H)=1$, by adding $\sqrt{n}$ random edges, one would modify $c$ by $O(1 / \sqrt{n})$ only, and a.a.s. induce at least one edge between a vertex of the cycle of type 1 vertices not yet in $H$ and a vertex of type 2 already in $H$, and sparsity and minimal rigidity remain true. So suppose no vertex is included in $H$, which is then also at least of size $\mu \varepsilon^{2} n$. Then by adding $\sqrt{n}$ random edges, one would modify $c$ by $O(1 / \sqrt{n})$ only, and a.a.s. induce at least three pairwise vertex-disjoint edges between the cycle of type 1 vertices and $H$. If we had $n_{1}(H) \geq 3$, keep one of the newly added edges and remove one edge of the cycle, if $n_{1}(H)=2$, keep one added edge, if $n_{1}(H)=1$, keep two edges, and if $n_{1}(H)=0$, keep three edges, without removing any edge in the last three cases. One can check that in all cases one obtains a minimally rigid block, as the total number of edges is correct and every subgraph is sparse. Hence we may assume 
that the cycle of type 1 vertices is included in $H$. Since the cycle of vertices of type 1 forms part of the 2.5-core, we know that the 2.5-core of $H$ is not empty. Then by Lemma 6.3, $H \subseteq K_{2.5}^{+}$. Now, by Theorem 2.20 and Remark 2.21 , we know that the size of $K_{2.5}^{+} / n$ tends to 0 when $c \rightarrow \frac{1}{1-q}$, and the second part of the statement of Theorem 2.11 follows.

\section{Proof of Theorem 2.16}

Proof. The proof is an easy generalization of [6], see [12]. We repeat the argument here for the convenience of the reader.

The 2.5-core of an arbitrary finite graph can be found by removing vertices of type 1 with degree $<2$ and vertices of type 2 with degree $<3$, in arbitrary order, until no such vertices exist. Let us call a vertex of type 1 with degree $<2$ or of type 2 with degree $<3$ a light vertex and let us call it a heavy vertex otherwise. We still obtain the 2.5-core by removing edges where one endpoint is light.

Regard each edge as consisting of two half-edges, each half-edge having one endpoint. We say that a half-edge is light or heavy when its endpoint is. As long as there is any light half-edge, choose one such half-edge uniformly at random and remove the edge it belongs to. When there are no light half-edges left, we stop. Then all light vertices are isolated and the heavy vertices with the remaining edges form the 2.5-core of the original graph.

We apply this algorithm to a random multigraph with given degree sequence $\left(d_{i}\right)_{1}^{n}$ (see [6], Section 2 for a precise definition). We observe the half-edges but not how they are connected into edges. At each step, we thus select a light half-edge at random. We then reveal its partner, which is random and uniformly distributed over the set of all other half-edges. We then remove these two half-edges and repeat as long as there is any light half-edge.

We now regard vertices as bins and half-edges as balls. Each bin inherits the type of its vertex. In each step, we remove first one random ball from the set of balls in light bins (i.e., bins of type 1 with $<2$ balls or bins of type 2 with $<3$ balls) and then a random ball without restriction. We stop when there are no non-empty light bins and the 2.5-core consists precisely of the heavy bins at the time we stop.

We thus alternately remove a random light ball and a random ball. We may just as well say that we first remove a random light ball. We then remove balls in pairs, first a random ball and then a random light ball, and stop with the random ball leaving no light ball to remove.

We now run this deletion process in continuous time such that, if there are $j$ balls remaining, then we wait an exponential time with mean $1 / j$ until the next pair of deletions. In other words, we make deletions at rate $j$. Let $L(t), H(t)$ denote the numbers of light and heavy balls at time $t$, respectively; further let $H_{1}(t)$ and $H_{2}(t)$ be the number of heavy bins of type 1 and 2 , respectively.

Let $\tau$ be the stopping time of this process. As in $[\underline{6}$, we first consider the total number of balls. This is a death process with rate 1 and jumps of size 2 , so that by Lemma 4.3 in [6], we have:

$$
\sup _{t \leq \tau}\left|L(t)+H(t)-2 m e^{-2 t}\right|=o_{p}(n) .
$$

We now concentrate on heavy balls. As shown in [6] (see Section 6), the same results can be applied if the degree sequence is not given, but converges in probability. In particular, the degree sequence of $G(n, c / n)$ is random, and it converges 
in probability to a Poisson distribution with mean $c$. Let $U_{r}^{1}(t)\left(\right.$ resp. $\left.U_{r}^{2}(t)\right)$ be the number of heavy bins of type 1 (resp. 2) with exactly $r$ balls at time t. Then by Lemma 4.4 in [6], we get (we use the fact that $\sum_{k \geq j} k e^{-\lambda} \frac{\lambda^{k}}{k !}=\lambda Q(\lambda, j-1)$ ):

$$
\begin{aligned}
\sup _{t \leq \tau}\left|\sum_{r \geq 2} r U_{r}^{1}(t) / n-(1-q) c e^{-t} Q\left(c e^{-t}, 1\right)\right| & =o_{p}(1) \\
\sup _{t \leq \tau}\left|\sum_{r \geq 3} r U_{r}^{2}(t) / n-q c e^{-t} Q\left(c e^{-t}, 2\right)\right| & =o_{p}(1)
\end{aligned}
$$

We define

$$
\begin{aligned}
h(x) & =(1-q) c x Q(c x, 1)+q c x Q(c x, 2) \\
h_{1}(x) & =(1-q) Q(c x, 2) \\
h_{2}(x) & =q Q(c x, 3) .
\end{aligned}
$$

Since we have $H(t)=\sum_{r} r U_{r}^{1}(t)+r U_{r}^{2}(t)$, we get:

$$
\begin{aligned}
& \sup _{t \leq \tau}\left|H(t) / n-h\left(e^{-t}\right)\right|=o_{p}(1) \\
& \sup _{t \leq \tau}\left|H_{1}(t) / n-h_{1}\left(e^{-t}\right)\right|=o_{p}(1) \\
& \sup _{t \leq \tau}\left|H_{2}(t) / n-h_{2}\left(e^{-t}\right)\right|=o_{p}(1)
\end{aligned}
$$

Hence we deduce that

$$
\sup _{t \leq \tau}\left|L(t) / n+h\left(e^{-t}\right)-c e^{-2 t}\right|=o_{p}(1) .
$$

In case (a), we have $c x^{2}-h(x)>0$ for all $x>0$, so that as in [6], we conclude that $\tau \rightarrow \infty$ a.a.s., $H(\tau) / n=H_{1}(\tau) / n=H_{2}(\tau) / n=o_{p}(1)$, and hence case (a) follows. In case (b), again following [6], we have $\tau \rightarrow-\log (\tilde{\xi} / c)$, and the claim follows.

\section{Proof of Theorem 2.20}

To prove Theorem 2.20, we need to prove that for a pair of vertices $a$ and $b$ chosen uniformly at random, we have:

$$
\operatorname{Pr}(a \text { in } 2.5+1.5 \text {-core })=(1+o(1))\left(1-e^{-\tilde{\xi}}-q \tilde{\xi} e^{-\tilde{\xi}}\right),
$$

and

(8) $\operatorname{Pr}(a$ and $b$ in $2.5+1.5$-core $) \leq(1+o(1))\left(1-e^{-\tilde{\xi}}-q \tilde{\xi} e^{-\tilde{\xi}}\right)^{2}$,

and the statement follows by Chebyshev's inequality.

To prove the first statement, we first consider the extended 2.5-core $C(a)$ obtained as in the previous section by removing vertices of type 1 with degree $<2$ and vertices of type 2 with degree $<3$ except for node $a$, that is, $a$ is considered as always heavy. Clearly the resulting graph contains the 2.5-core. Note that if we condition the resulting graph on its degree sequence, it is still a configuration model (see Theorems 10 and 11 in [12]).

We have:

(i) if $a$ is of type 1 (resp. 2) and has degree 0 (resp. 0 or 1) in $C(a)$, then $a$ is not in the $2.5+1.5$-core. 
(ii) if $a$ is of type 1 (resp. 2) and has degree $\geq 2$ (resp. $\geq 3$ ) in $C(a)$, then $a$ is in the 2.5-core.

(iii) if $a$ is of type 1 (resp. 2) and has degree 1 (resp. 2) in $C(a)$, then we can remove $a$ and then continue the algorithm by removing vertices of type 1 with degree $<2$ and vertices of type 2 with degree $<3$ to get the 2.5 -core.

In the case of (iii), if the graph induced by $a$ and the nodes removed during this second phase is a tree, then it follows that $a$ is part of the $2.5+1.5$-core. Note that as long as the number of nodes removed during this second phase is $o\left(n^{1 / 3}\right)$, the graph induced by $a$ and the removed nodes is a tree w.h.p.: for each node the probability to connect to one of the $o\left(n^{1 / 3}\right)$ nodes is $o\left(n^{-2 / 3} \log n\right)$, and by a union bound over all nodes the desired result follows.

We clearly have

$$
\operatorname{Pr}(a \text { in } 2.5+1.5 \text {-core }) \leq \operatorname{Pr}(a \text { has degree at least } t(a) \text { in } C(a)),
$$

where $t(a) \in\{1,2\}$ is the type of $a$. Hence, we need to compute the probability that $a$ has at least 1 neighbor in $C(a)$ in the case of being of type 1 (at least 2 neighbors in $C(a)$ in the case of being of type 2$)$. When changing only one vertex $a$ to be always heavy, the functions $h(x), h_{1}(x), h_{2}(x)$ change only by an additive $O(\log n / n)$, and thus $\tilde{c}(q)$ and $\tilde{\xi}(q)$ also change by at most an additive $o(1)$. Hence, for $c>\tilde{c}(q)$, as in the previous proof, for the stopping time $\tau$ we still have $\tau \sim-\log (\tilde{\xi} / c)$. Therefore, we have to compute the probability that at time $\tau, a$ has at least 1 neighbor in $C(a)$ in the case of being of type 1 (at least 2 in the case of being of type 2). Note that since $a$ is heavy, the probability for each halfedge incident to $a$ to be alive at time $t$ is $e^{-t}$. Since $a$ is chosen uniformly at random, we have

$$
\begin{gathered}
\operatorname{Pr}(a \text { has degree at least } t(a) \text { in } C(a)) \\
=\quad(1+o(1)) \sum_{k \geq 0}\left(\frac{e^{-c} c^{k}}{k !}\left((1-q)\left(1-\left(1-e^{-\tau}\right)^{k}\right)+q\left(1-\left(1-e^{-\tau}\right)^{k}-k e^{-\tau}\left(1-e^{-\tau}\right)^{k-1}\right)\right)\right) \\
= \\
(1+o(1))\left(1-e^{-\tilde{\xi}}-q \tilde{\xi} e^{-\tilde{\xi}}\right) .
\end{gathered}
$$

We now prove (8). Consider two special vertices $a$ and $b$, chosen uniformly at random, and consider them both heavy. By the same reasoning as above, the functions $\tilde{c}(q), \tilde{\xi}(q), h(x), h_{1}(x), h_{2}(x)$ change only by additive terms of $o(1)$. Again, note that in order for both $a$ and $b$ to be in the $2.5+1.5$-core, it is necessary but not sufficient for both $a$ and $b$ to have at time $\tau$ still 1 incident edge in the case of being of type 1 ( 2 incident edges in the case of being of type 2 ). Hence,

$$
\begin{gathered}
\operatorname{Pr}(a \text { and } b \text { in } 2.5+1.5 \text {-core }) \\
\leq \quad(1+o(1))\left(1-e^{-\tilde{\xi}}-q \tilde{\xi} e^{-\tilde{\xi}}\right)^{2} .
\end{gathered}
$$

We now prove that $(9)$ is tight. First, we compute the degree distribution in $C(a)$. We use the same notation as in the proof of Theorem 2.16. It follows from Lemma 4.4 in [6] (see also Lemma 12 in [12]) that we have

$$
\begin{aligned}
\frac{U_{r}^{1}(t)}{n} & =(1-q) \frac{\left(c e^{-t}\right)^{r} e^{-c e^{-t}}}{r !}+o(1), \quad r \geq 2 \\
\frac{U_{r}^{2}(t)}{n} & =q \frac{\left(c e^{-t}\right)^{r} e^{-c e^{-t}}}{r !}+o(1), \quad r \geq 3 .
\end{aligned}
$$


In particular, at $\tau=-\log (\tilde{\xi} / c)+o(1)$, the stopping time of the process, we have

$$
\begin{aligned}
\frac{U_{r}^{1}(\tau)}{n} & =(1-q) \frac{\tilde{\xi}^{r} e^{-\tilde{\xi}}}{r !}+o(1), \quad r \geq 2 \\
\frac{U_{r}^{2}(\tau)}{n} & =q \frac{\tilde{\xi}^{r} e^{-\tilde{\xi}}}{r !}+o(1), \quad r \geq 3 .
\end{aligned}
$$

As computed above, the total number of half-edges is

$$
\sum_{r \geq 2} r U_{r}^{1}(\tau)+\sum_{r \geq 3} r U_{r}^{2}(\tau)=n h(\tilde{\xi} / c)+o(n)=n \frac{\tilde{\xi}^{2}}{c}+o(n) .
$$

Hence, when choosing a ball uniformly at random among the balls in bins corresponding to heavy vertices, the probability to pick a node of type 1 with degree 2 is

$$
p_{2}^{1}=\frac{2 U_{2}^{1}(\tau)}{\sum_{r} r U_{r}^{1}(\tau)+r U_{r}^{2}(\tau)}=(1-q) e^{-\tilde{\xi}} c+o(1),
$$

and similarly, the probability to pick a node of type 2 with degree 3 is

$$
p_{3}^{2}=\frac{3 U_{3}^{2}(\tau)}{\sum_{r} r U_{r}^{1}(\tau)+r U_{r}^{2}(\tau)}=q \frac{\tilde{\xi} c}{2} e^{-\tilde{\xi}}+o(1) .
$$

Consider a new node of type $t \in\{1,2\}$, picked up during the second phase of the algorithm in case (iii); as long as the neighborhood of $a$ explored in this second phase is a tree, this new node has at least $t+1$ half-edges, since it is heavy. If it has exactly $t+1$ half-edges, it becomes light after removal of one half-edge and the algorithm continues: a type 1 node then induces one more half-edge to remove, and a type 2 node two more half-edges. So to show that this branching exploration process is $o\left(n^{1 / 3}\right)$, it suffices to prove that it is subcritical, that is,

$$
2 p_{3}^{2}+p_{2}^{1}<1
$$

and this will imply that $(9)$ is indeed tight. We have

$$
2 p_{3}^{2}+p_{2}^{1}=q \tilde{\xi} c e^{-\tilde{\xi}}+(1-q) e^{-\tilde{\xi}} c+o(1),
$$

and we will now show that this is indeed less than 1 for $c>\tilde{c}(q)$. Recall that for $c>\tilde{c}(q)$ (the case of interest here), $\tilde{\xi}(c, q)>0$ is the largest solution of the equation

$$
c=\psi(\xi ; q) \quad \text { with } \quad \psi(\xi ; q)=\frac{\xi}{1-e^{-\xi}-q \xi e^{-\xi}} .
$$

Then, necessarily at the point $(\tilde{\xi}(c, q), q)$ :

$$
\frac{\partial \psi}{\partial \xi}=\frac{e^{\xi}\left(-q \xi^{2}-\xi+e^{\xi}-1\right)}{\left(q \xi-e^{\xi}+1\right)^{2}} \geq 0,
$$

as otherwise 10 would have a larger solution (note that $\psi(\xi ; q) \rightarrow \infty$ as $\xi \rightarrow \infty$ ). Furthermore, if $q \leq 1 / 2, \partial \psi / \partial \xi$ has no strictly positive root, and in this case, for $c>\tilde{c}(q), \frac{\partial \psi}{\partial \xi}>0$ at the point $(\tilde{\xi}(c, q), q)$. Now, if $q>1 / 2$, note that $\partial \psi / \partial \xi$ has a single strictly positive root: indeed, for $\xi>0, \partial \psi / \partial \xi(\xi)=0$ iff $g(\xi):=$ $e^{\xi}-1-q \xi^{2}-\xi=0$. Now, $g^{\prime}(\xi)=e^{\xi}-1-2 q \xi$ and $g^{\prime \prime}(\xi)=e^{\xi}-2 q$, and hence $g(\xi)$ is convex for $\xi \geq \log (2 q)>0$ and concave otherwise. Also, $g(0)=g^{\prime}(0)=0$, and since $g^{\prime \prime}(0)<0$, the function is first decreasing, and then increasing with $g(\xi) \rightarrow \infty$ as $\xi \rightarrow \infty$, therefore passing exactly once by 0 , giving the single strictly positive 
root. This root is a minimum of $\psi$ and equal to $\tilde{\xi}(\tilde{c}(q), q)$ : recall the definition of $\tilde{c}(q)$

$$
\tilde{c}(q)=\inf _{\xi>0} \psi(\xi ; q) .
$$

Hence for $c>\tilde{c}(q), \partial \psi / \partial \xi$ cannot vanish at the point $(\tilde{\xi}(c, q), q)$, thus it is strictly positive. Using (11), this is equivalent to

$$
e^{-\tilde{\xi}}\left(1+\tilde{\xi}+q \tilde{\xi}^{2}\right)<1
$$

and therefore

$$
\tilde{\xi}\left(q e^{-\tilde{\xi}} \tilde{\xi}+(1-q) e^{-\tilde{\xi}}\right)<(1-q)\left(1-e^{-\tilde{\xi}}\right)+q\left(1-e^{-\tilde{\xi}}-\tilde{\xi} e^{-\tilde{\xi}}\right) .
$$

Thus, the expression $2 p_{3}^{2}+p_{2}^{1}$ at $c>\tilde{c}(q)$ is

$$
\frac{\tilde{\xi} e^{-\tilde{\xi}}(q \tilde{\xi}+(1-q))}{(1-q)\left(1-e^{-\tilde{\xi}}\right)+q\left(1-e^{-\tilde{\xi}}-\tilde{\xi} e^{-\tilde{\xi}}\right)}<1,
$$

as desired.

The result follows.

\section{Proof of Lemma 2.22}

In order to prove Lemma 2.22, we first need to prove the following auxiliary lemma:

Lemma 9.1. Let $\alpha \leq 1$. Let $r, s, t=\alpha s \in \mathbb{N}$ and let $s_{1}, \ldots, s_{r} \in \mathbb{N}$ such that $\sum_{i=1}^{r} s_{i}=\alpha s$ and $\sum_{i=1}^{r} i s_{i}=s$. There exists $C>0$ such that for any $r>0$, and any $\alpha$ we have

$$
\prod_{i=1}^{r} \frac{1}{s_{i}^{s_{i}}} \leq C^{\alpha s} /\left(\alpha^{2} s\right)^{\alpha s}
$$

Proof. It is sufficient to prove

$$
\sum_{i=1}^{r} s_{i} \log s_{i} \geq \alpha s \log \alpha s+\alpha s \log \alpha-\alpha s \log C
$$

We first normalize $s_{i}$. Writing $\tilde{s}_{i}=s_{i} /(\alpha s)$, the constraints are

$$
\sum_{i=1}^{r} \tilde{s}_{i}=1 \quad ; \quad \sum_{i=1}^{r} i \tilde{s}_{i}=\frac{1}{\alpha}
$$

We will show that we can find $C>0$ independent of $r$ and $\alpha$ such that

$$
\sum_{i=1}^{r} \tilde{s}_{i} \log \tilde{s}_{i} \geq \log \alpha-\log C
$$

To simplify notation, we write simply $s_{i}$ from now on.

For a fixed $r$, we maximize $\sum_{i=1}^{r}-s_{i} \log s_{i}$ subject to $\sum_{i=1}^{r} s_{i}=1$ and $\sum_{i=1}^{r} i s_{i}=$ $1 / \alpha$. Applying Lagrange multipliers gives the system of equations

$$
\log s_{i}+1+\lambda_{1}+i \lambda_{2}=0, \quad i=1, \ldots, r,
$$

and thus an optimal solution has to satisfy $s_{i}=a b^{i}$ for some $a, b \in \mathbb{R}$. It is enough to show 12 for all $s_{i}$ of this form. 
ON RIGIDITY, ORIENTABILITY AND CORES OF RANDOM GRAPHS WITH SLIDERS 25

The two constraints translate into the two following equations, repeatedly used in the following:

$$
\begin{aligned}
a & =\frac{1-b}{b\left(1-b^{r}\right)} \\
\frac{1-(r+1) b^{r}+r b^{r+1}}{(1-b)\left(1-b^{r}\right)} & =\frac{1}{\alpha}
\end{aligned}
$$

We distinguish five cases.

Case 1: there exists $K>0$ such that $1 / \alpha \geq r / K$.

In this case, we may ignore the constraint $\sum_{i=1}^{r} i s_{i}=1 / \alpha$. Clearly, $\sum_{i=1}^{r} s_{i} \log s_{i}$ is minimized by the uniform distribution $s_{i}=1 / r$. Hence

$$
\sum_{i=1}^{r} s_{i} \log s_{i} \geq-\log r \geq \log \alpha-\log K
$$

and we are done.

Case 2: $r<R_{0}$, where $R_{0}$ is some large enough constant such that $(r-1)(1 / 2)^{r+1}<$ 0.5 for all $r \geq R_{0}$.

Reasoning as in the previous case, we obtain

$$
\sum_{i=1}^{r} s_{i} \log s_{i} \geq-\log R_{0}
$$

and we are done as well.

Case 3: $b \geq 1$.

Note that we always have

$$
\sum_{i=\lfloor r / 2\rfloor}^{r} s_{i} \leq \frac{\sum_{i=1}^{r} i s_{i}}{\lfloor r / 2\rfloor}
$$

If $b \geq 1, s_{i}$ is increasing, thus the left hand side is larger than $1 / 2$. Hence $(1 / \alpha)=$ $\sum_{i=1}^{r} i s_{i} \geq\lfloor r / 2\rfloor / 2 \geq r / 5$. We may choose $K=5$ and apply Case 1 .

Case 4: $b \leq 1 / 2$, and $r \geq R_{0}$.

We have

$$
a=\frac{1}{b\left(1+b+\ldots+b^{r-1}\right)}=\frac{1}{b(1+c(b) b)}
$$

with $1 \leq c(b) \leq 2$. Using $\sum_{i=1}^{r} s_{i} \log s_{i}=\sum_{i=1}^{r} s_{i}(\log a+i \log b)=\log a+\frac{1}{\alpha} \log b$ we obtain

$$
\sum_{i=1}^{r} s_{i} \log s_{i}=\log a+\frac{1}{\alpha} \log b=(1 / \alpha-1) \log b-\log (1+c(b) b) \geq\left(\frac{1}{\alpha}-1\right) \log b-\log 2 .
$$

Using that $(1-b)\left(1-b^{r}\right) \geq \frac{1}{4}$, we get

$$
\begin{aligned}
\frac{1}{\alpha} & =\frac{1-(r+1) b^{r}+r b^{r+1}}{(1-b)\left(1-b^{r}\right)} \\
\frac{1}{\alpha}-1 & =\frac{b-r b^{r}+(r-1) b^{r+1}}{(1-b)\left(1-b^{r}\right)} \\
& \leq 4\left(b+(r-1) b^{r+1}\right) \\
& \leq 4 b(1+0.5)=6 b,
\end{aligned}
$$


where we used that for $R \geq R_{0}$, we have $(r-1) b^{r+1} \leq(r-1)(1 / 2)^{r+1} \leq 0.5$. Thus,

$$
\left(\frac{1}{\alpha}-1\right) \log b \geq 6 b \log b \geq C
$$

since $6 b \log b$ is for $b \in[0,1 / 2]$ bounded below by an absolute constant. Combining this last estimate with $(13)$, the desired inequality holds.

Case 5: $1 / 2 \leq b<1$ and $1 / \alpha \leq r / K$.

Using $\log a=\log (1-b)-\log b-\log \left(1-b^{r}\right) \geq \log (1-b)$, we obtain

$$
\sum_{i=1}^{r} s_{i} \log s_{i}=\log a+\frac{1}{\alpha} \log b \geq \log (1-b)+\frac{1}{\alpha} \log b .
$$

We will show that $\log b / \alpha$ is bounded from below by some constant, and that $\log (1-b) \geq \log \alpha+C$. First note that we have the following relation:

$$
\frac{1-b}{\alpha}=1-r \frac{b^{r}(1-b)}{1-b^{r}}
$$

Consider first $\log b / \alpha$. If $\alpha \geq 1 / 2$, then $\log b / \alpha \geq 2 \log b \geq-2 \log 2$, and we have the desired bound. Assume then $\alpha<1 / 2$. From (14), we have $(1-b) / \alpha \leq 1$, and thus, $\log b \geq \log (1-\alpha)$. Also, since $\alpha<1 / 2, \log (1-\alpha) \geq-2 \alpha$. Hence,

$$
\frac{\log b}{\alpha} \geq-2
$$

and we are done with this term. Let us turn to the $\log (1-b)$ term. For any $i$, we have $s_{i} \geq a b^{r}$, and hence

$$
\frac{r}{K} \geq \frac{1}{\alpha}=\sum_{i=1}^{r} i s_{i} \geq a b^{r} \frac{r(r+1)}{2}
$$

Thus

$$
\frac{2 b}{K} \geq(r+1) \frac{b^{r}(1-b)}{1-b^{r}}
$$

and since $b r /(r+1) \leq 1$, also

$$
\frac{2}{K} \geq r \frac{b^{r}(1-b)}{1-b^{r}}
$$

Inserting this into (14), we have

$$
\frac{1-b}{\alpha} \geq 1-\frac{2}{K}
$$

Thus $\log (1-b) \geq \log \alpha+C$, and we are done.

We need the following two lemmas.

Lemma 9.2. 2] [Corollary 5.8]

Let $G \in \mathcal{G}(n, p)$ with $p=c / n$ and $0<c<1$. The following holds a.a.s.:

- $G$ contains only trees and unicyclic components.

- The number of vertices in unicyclic components is at most $\omega_{n}$, for some arbitrarily slowly growing function $\omega_{n}$.

The following lemma can easily be derived from Corollary 5.11 and Theorem 5.5 of [2], by extending it to non-constant values of $k$ : 
Lemma 9.3. Let $G \in \mathcal{G}(n, p)$ with $p=c / n$ and $0<c<1$. Let $T_{k}$ be the number of trees of size $k$ in $G$. Let $\omega_{n}$ be an arbitrarily slowly growing function with $n$. The following holds a.a.s.:

- $T_{k}=0$ for $k=\omega(\log n)$.

- $T_{1}=n e^{-c}(1+o(1))$.

- For any $2 \leq k=O(\log n)$ with $\frac{n k^{k-2}}{k !} c^{k-1} e^{-k c} \geq \omega_{n}, T_{k}=\frac{n k^{k-2}}{k !} c^{k-1} e^{-k c}(1+$ $o(1))$.

We need one more lemma, in the spirit of Theorem 5.10 of [2].

Lemma 9.4. Let $G \in \mathcal{G}(n, p)$ with $p=c / n$ and $0<c<1$. Let $\eta>0$ be a sufficiently small constant. Let $\omega_{n}$ be any function growing with $n$ arbitrarily slowly, and let $k_{0}:=\lfloor\nu \log n\rfloor$ be the smallest integer $k$ satisfying $\eta \omega_{n} \leq \mathbb{E}\left(T_{k}\right)=$ $\frac{n k^{k-2}}{k !} c^{k-1} e^{-k c} \leq \omega_{n}$ (since $0<c<1$, such an integer must exist for sufficiently small $\eta$ ). Then there exists a constant $C>0$ such that a.a.s. $\sum_{k \geq k_{0}} T_{k} \leq C \mathbb{E}\left(T_{k_{0}}\right)$.

Proof. Clearly, $\mathbb{E}\left(T_{k_{0}}\right) \leq \sum_{k \geq k_{0}} \mathbb{E}\left(T_{k}\right)$. By Stirling's formula,

$$
\begin{aligned}
\sum_{k \geq k_{0}} \mathbb{E}\left(T_{k}\right) & =(1+o(1))\left(c e^{1-c}\right)^{k_{0}} \frac{n}{\sqrt{2 \pi} c} \sum_{i \geq 0} \frac{\left(c e^{1-c}\right)^{i}}{\left(i+k_{0}\right)^{2.5}} \\
& \leq(1+o(1))\left(c e^{1-c}\right)^{k_{0}} \frac{n}{\sqrt{2 \pi} c k_{0}^{2.5}} \sum_{i \geq 0}\left(c e^{1-c}\right)^{i} \\
& =\mathbb{E}\left(T_{k_{0}}\right) \frac{1}{1-\left(c e^{1-c}\right)^{i}} .
\end{aligned}
$$

Writing $T_{k}$ as a sum of indicator variables over all $k$-tuples of vertices, we see that when considering two disjoint trees of size at most $O(\log n)$, at most $O(\log n)$ non-edges incident to each vertex of the second tree are exposed when given the first. Hence, the probability of having no edge adjacent to any of the vertices changes only by a factor $(1+o(1))$, and thus $\mathbb{E}\left(\left(\sum_{k \geq k_{0}} T_{k}\right)^{2}\right)=2 \mathbb{E}\left(\sum_{\ell>k \geq k_{0}} T_{k} T_{\ell}\right)+$ $\mathbb{E}\left(\sum_{k \geq k_{0}} T_{k}^{2}\right)=(1+o(1)) \sum_{k \geq k_{0}} \mathbb{E}\left(T_{k}\right) \sum_{\ell \geq k_{0}} \mathbb{E}\left(\bar{T}_{\ell}\right)=(1+o(1))\left(\sum_{k \geq k_{0}} \mathbb{E}\left(T_{k}\right)\right)^{2}$. By Chebyshev's inequality, the result follows.

We turn now to the proof of Lemma 2.22

Proof. For a subgraph of size $u$, let $n_{1}$ its number of vertices of type 1 and $n_{2}$ its number of vertices of type 2 (we do not explicitly refer to the size nor to the subgraph, since it is clear from the context). Let $X_{u}$ denote the number of subgraphs of size $u \leq \alpha n$ with more than $n_{1}+2 n_{2}$ edges. Our goal is to show that for a randomly chosen graph $G \in \mathcal{G}(n, p)$ we have $\sum_{u \leq \alpha n} X_{u}=0$ a.a.s. To simplify the notation, we set $r=1-q$. Let $\omega_{n}$ denote a function tending to infinity arbitrarily slowly, as $n \rightarrow \infty$.

We start with the relatively easy cases where $u$ is small enough, or $n_{2}$ large enough.

Small $u: u=o(\log n / \log \log n)$

First note that the expected number of subgraphs of size $u=o(\log n / \log \log n)$ 
with at least $n_{1}+2 n_{2}+1$ edges is at most

$$
\begin{aligned}
& \left.\sum_{n_{1}=0}^{\log n /\left(\omega_{n} \log \log n\right) \log n /\left(\omega_{n} \log \log n\right)} \sum_{n_{2}=0}^{r n(1+o(1))} \begin{array}{c}
q n(1+o(1)) \\
n_{1}
\end{array}\right)\left(\begin{array}{c}
\left(\begin{array}{c}
n_{1}+n_{2} \\
2
\end{array}\right) \\
n_{2}+2 n_{2}+1
\end{array}\right) p^{n_{1}+2 n_{2}+1} \\
\leq & \sum_{n_{1}=0}^{\log n /\left(\omega_{n} \log \log n\right) \log n /\left(\omega_{n} \log \log n\right)}\left(\frac{r n e}{n_{1}}\right)^{n_{1}}\left(\frac{q n e}{n_{2}}\right)^{n_{2}}\left(\frac{c e\left(n_{1}+n_{2}\right)^{2}}{2 n\left(n_{1}+2 n_{2}+1\right)}\right)^{n_{1}+2 n_{2}+1} \\
\leq & \sum_{n_{2}=0}^{\log n /\left(\omega_{n} \log \log n\right) \log n /\left(\omega_{n} \log \log n\right)}\left(\frac{1}{n}\right)^{n_{2}+1}\left(O\left(n_{1}+n_{2}\right)\right)^{n_{1}+2 n_{2}+1} \\
\leq & \frac{\log ^{2} n}{n}(O(\log n))^{\left(2 \log n / \omega_{n} \log \log n\right)}=o(1),
\end{aligned}
$$

and thus a.a.s. there is no such subgraph.

$$
\text { Large } n_{2}: n_{2}>\xi n_{1}
$$

Also, a subgraph with more than $n_{1}+2 n_{2}+1$ edges and total size at most $\alpha n$ cannot exist, if the density of nodes of type 2 is too big: more precisely, if $n_{2}>\xi n_{1}$ for some constant $\xi>0$, then by Proposition 4 of [19] applied with $a=\frac{1+2 \xi}{1+\xi}>1$, there exists a constant $t(a, c)=\left(\frac{2 a}{c}\right)^{a /(a-1)} e^{-(a+1) /(a-1)}$, such that a.a.s. $G(n, c / n)$ has no subgraph with $n_{1}+2 n_{2}$ edges of size at most $t(a, c) n$.

\section{Remaining cases: $u=\Omega(\log n / \log \log n)$ and $n_{2} \leq n_{1} / K$}

We may thus assume that we are dealing with the remaining cases of $u=\Omega(\log n / \log \log n)$ and $n_{2} \leq n_{1} / K$. We drop the condition $u \leq \alpha n$ from now on.

Let $K^{\prime}=K^{\prime}(c-1 / r)$ the constant coming from part 2 of Lemma 9.4 so that a.a.s. all trees are of size at most $K^{\prime} \log n$, and let $K=K\left(K^{\prime}\right)$ be a sufficiently large constant (in fact, $K$ depends on $q$ and $c$, and it is the largest constant appearing throughout this proof; in particular, we choose $K$ to be such that it is also larger than the product of $1 / r c$ and the constants $C$ of the statement of Lemma 9.1 and the constant $C$ of the statement of Lemma 9.4.

We need one more observation: when counting all components $X_{u}$ with more than $n_{1}+2 n_{2}$ edges, let us first choose the components from the subgraph induced by the $n_{1}$ vertices. We may assume that each of these components is taken entirely or not at all: indeed, since $p=c / n$ with $c<1 / r$, and since the probability for an edge to be present in the subgraph induced by all $r n(1+o(1))$ vertices of type 1 is $p=c / n=\frac{c r}{r n}<\frac{1}{r n}$, for the subgraph induced by all $r n(1+o(1))$ vertices of type 1, Lemma 9.2 applies (with $c r$ playing the role of $c$, and $r n$ playing the role of $n$ ), and thus this subgraph a.a.s. contains only trees and unicyclic components. If there were now a subgraph with $n_{1}$ vertices of type 1 and $n_{2}$ vertices of type 2 and more than $n_{1}+2 n_{2}$ edges, where some components are partially taken, we could complete the tree components and unicyclic components. We add at least the same number of edges as vertices, and the resulting subgraph would have $n_{1}^{\prime}$ vertices of type $1, n_{2}$ vertices of type 2 (clearly still satisfying $n_{2} \leq n_{1}^{\prime} / K$ ), and it would still have more than $n_{1}^{\prime}+2 n_{2}$ edges. Thus we will from now on count only subgraphs where all components of the subgraph induced by the vertices of type 1 are entirely or not at all taken. 
Denote by $T_{i}$ the number of trees of size $i$ of this subgraph induced by the vertices of type 1. By Lemma 9.3 , a.a.s., $T_{1}=r n e^{-r c}(1+o(1)) \leq\left(r n r c e^{1-r c}\right) \frac{1}{r c}$, and using Stirling's formula, for each $2 \leq i=O(\log n)$ satisfying $\frac{r n i^{i-2}}{i !}(r c)^{i-1} e^{-i r c} \geq$ $\omega_{n}$, a.a.s., $T_{i}=(1+o(1)) \frac{r n i^{i-2}}{i !}(r c)^{i-1} e^{-i r c} \leq r n\left(r c e^{1-r c}\right)^{i} \frac{1}{r c}$. For the remaining number of trees of size $i=\Theta(\log n)$, let $i_{0}:=\lfloor\nu \log n\rfloor$ throughout the proof be the smallest integer $i$ satisfying

$$
\eta \omega_{n} \leq \mathbb{E}\left(T_{i}\right)=\frac{r n i^{i-2}}{i !}(r c)^{i-1} e^{-i r c}=(1+o(1)) \frac{r n\left(r c e^{1-r c}\right)^{i}}{\sqrt{2 \pi} r c i^{2.5}} \leq \omega_{n}
$$

for some sufficiently small $\eta>0$ (as remarked before, such $i_{0}$ exists for small enough $\eta>0)$. By the proof of Lemma 9.4 the number of trees of size at least $i_{0}$ is a.a.s. at most $(1+o(1)) \frac{1}{1-c r e^{1-c r}} \mathbb{E}\left(T_{i_{0}}\right) \leq(1+o(1)) \frac{1}{1-c r e^{1-c r}} r n\left(r c e^{1-r c}\right)^{i_{0}}$. Finally, once more by Lemma 9.3 , the total number of trees of size $i=\omega(\log n)$ is 0 a.a.s.

Consider now any graph on $n$ vertices, for which the subgraph induced by $(1+$ $o(1)) r n$ vertices (that will then correspond to vertices of type 1) is deterministically given: it consists only of trees and unicyclic components, the number of trees of size $i<i_{0}$ is at most $r n\left(r c e^{1-r c}\right)^{i} \frac{1}{r c}$, the total number of trees of size $i=\Theta(\log n)$ for $i \geq i_{0}$ is at most $(1+o(1)) \frac{1}{1-c r e^{1-c r}} r n\left(r c e^{1-r c}\right)^{i_{0}}$, the number of trees of size $\omega(\log n)$ is 0 , and the number of vertices in unicyclic components is at most $\omega_{n}$. We will below show that starting with any such graph, when exposing the random edges between the $q n$ vertices of type 2 and edges between type 1 and type 2 (as before, each such edge being present with probability $p$ ), with probability $1+o(1)$ in the whole graph there are no subgraphs $X_{u}, u \leq \alpha n$ with more than $n_{1}+2 n_{2}$ edges. The lemma will then follow, since the randomly chosen graph $G \in \mathcal{G}(n, p)$, as shown above, a.a.s. satisfies these properties.

It remains now to show that any graph on $(1+o(1)) r n$ vertices (of type 1 ) with the above mentioned properties has a.a.s. no subgraphs with more than $n_{1}+2 n_{2}$ edges. By definition, we can bound the number of such components of each size in the subgraph induced by the vertices of type 1 . We then have to combine them with the choices for the $n_{2}$ vertices. If there are $t$ tree components in the subgraph induced by the vertices of type 1 , the number of additional edges needed to surpass $n_{1}+2 n_{2}$ is $2 n_{2}+t+1$. Call tree components of size $i<i_{0}$ to be small, and call trees of size $i_{0} \leq i=\Theta(\log n)$ to be medium. For any subgraph with $n_{1}=\Omega(\log n / \log \log n)$ vertices of type 1 , write $n_{1}=n_{t}+z$, where $n_{t}$ is the number of vertices belonging to tree components and $z$ is the number of vertices belonging to unicyclic components. Note that $z \leq \omega_{n}$ and that by our assumption on $n_{1}, z=o\left(n_{1}\right)$. Next, for small trees of size $i<i_{0}$, let $s_{i}$ be the number of tree components of size $i$ in the subgraph of the $n_{1}$ vertices, and denote by $s_{i_{0}}$ the number of medium tree components (of size at least $\left.i_{0}\right)$ in the subgraph of the $n_{1}$ vertices. Let $\sum_{i=1}^{i_{0}} i s_{i}=s$, and let $\sum_{i=1}^{i_{0}} s_{i}=\alpha s$ for $\alpha \leq 1$. Note that $s$ is therefore a lower bound on the number of vertices in trees in the subgraph of the $n_{1}$ vertices; also, note that all medium sized trees are of size $\Theta(\log n)$, and therefore $s \geq n_{1} / K^{\prime}$ and still $z=o(s)$.) Define now $X_{u}^{s, \alpha s, n_{1}, n_{2}}$ the number of subgraphs $X_{u}$ with $n_{1}$ vertices of type $1, n_{2}$ vertices of type 2 , satisfying $\sum_{i=1}^{i_{0}} i s_{i}=s$, and $\sum_{i=1}^{i_{0}} s_{i}=\alpha s$, furthermore having at most $\omega_{n}$ vertices in unicyclic components and having in total more than $n_{1}+2 n_{2}$ edges (the previously imposed restrictions $s \geq n_{1} / K^{\prime}, n_{2} \leq n_{1} / K$ and $n_{1}=\Omega(\log n / \log \log n)$ still hold). We have for some large constants $C, C^{\prime}, C^{\prime \prime}>0$ (whose values might 
change from line to line)

$$
\begin{aligned}
\mathbb{E}\left(X_{u}^{s, \alpha s, n_{1}, n_{2}}\right) & \leq \sum_{\sum s_{i}=\alpha s, \sum_{i} i s_{i}=s}\left(2^{\omega_{n}} \prod_{i}\left(\begin{array}{c}
r n\left(r c e^{1-r c}\right)^{i} C \\
s_{i}
\end{array}\right)\left(\begin{array}{c}
q n(1+o(1)) \\
n_{2}
\end{array}\right)\left(\begin{array}{c}
n_{1} n_{2}+\left(\begin{array}{c}
n_{2} \\
2
\end{array}\right) \\
2 n_{2}+\sum s_{i}
\end{array}\right) p^{2 n_{2}+\sum s_{i}+1}\right) \\
& \leq 2^{\omega_{n}}\left(C^{\prime} n\right)^{\alpha s}\left(r c e^{1-r c}\right)^{s}\left(\frac{q e n(1+o(1))}{n_{2}}\right)^{n_{2}}\left(\frac{\left(C^{\prime \prime} s n_{2}+\frac{1}{2} n_{2}^{2}\right) e c}{\left(2 n_{2}+\alpha s\right) n}\right)^{2 n_{2}+\alpha s} p \prod_{i} \frac{1}{s_{i}^{s_{i}}} .
\end{aligned}
$$

By Lemma 9.1. $\prod_{i} \frac{1}{s_{i}} \leq\left(\frac{C}{\alpha^{2} s}\right)^{\alpha s}$ for some $C>0$, and writing $n_{2}=\beta \alpha s$ (note that since $n_{2} \leq n_{1} / K$ and $s \geq n_{1} / K^{\prime}$, we still have $\beta \alpha \leq K^{\prime} / K$, which is still sufficiently small for large enough $K=K\left(K^{\prime}\right)$ ), we have (again for large enough $\left.C, C^{\prime}>0\right)$

$$
\begin{aligned}
2^{-\omega_{n}} \mathbb{E}\left(X_{u}^{s, \alpha s, n_{1}, n_{2}}\right) & \leq\left(\frac{C^{\alpha} r c e^{1-r c}\left(\beta \alpha s^{2}\right)^{\alpha}}{\left.\left(\alpha^{2} s\right)^{\alpha}(2 \beta \alpha s+\alpha s)^{\alpha}\right)}\right)^{s}\left(\frac{C\left(\beta \alpha s^{2}\right)^{2}}{\beta \alpha s(2 \beta \alpha s+\alpha s)^{2} n}\right)^{\beta \alpha s} \\
& \leq\left(\frac{C^{\prime \alpha} r c e^{1-r c} \beta^{\alpha}}{\alpha^{2 \alpha}(1+2 \beta)^{\alpha}} \frac{(C \beta s)^{\beta \alpha}}{(1+2 \beta)^{2 \beta \alpha}(\alpha n)^{\beta \alpha}}\right)^{s} .
\end{aligned}
$$

We distinguish now three cases. If $\beta \geq K^{\prime} / K^{3 / 4}$, then $\alpha \leq 1 / K^{1 / 4}$. Then, for some $C^{\prime \prime}>0$, we have

$$
2^{-\omega_{n}} \mathbb{E}\left(X_{u}^{s, \alpha s, n_{1}, n_{2}}\right) \leq\left(\frac{\left(r c e^{1-r c}\right) C^{\prime \prime \alpha}(C s)^{\beta \alpha}}{\alpha^{2 \alpha} \beta^{\beta \alpha}(\alpha n)^{\beta \alpha}}\right)^{s} .
$$

The base of the last expression is clearly monotone increasing in $s$, and so we may plug in our upper bound on $s \leq u \leq n$. Considering the base only and taking logarithms, we obtain

$$
\log \left(r c e^{1-r c}\right)-2 \alpha \log \alpha-\beta \alpha \log (\beta \alpha)+\alpha \log C^{\prime \prime}+\beta \alpha \log C .
$$

Note that $x \log x \rightarrow 0$ as $x \rightarrow 0$, and thus, if $K$ and thus also $K^{1 / 4}$ is sufficiently large, both $\alpha$ and $\beta \alpha$ are sufficiently small. Then the first term dominates in absolute value, and since $r c e^{1-r c}<1$, the expression is negative.

If $\beta<K^{\prime} / K^{3 / 4}$ and $\alpha \leq 1 / K^{1 / 4}$, then for some $C^{\prime \prime}>0$

$$
2^{-\omega_{n}} \mathbb{E}\left(X_{u}^{s, \alpha s, n_{1}, n_{2}}\right) \leq\left(\frac{\left(r c e^{1-r c}\right) C^{\prime \prime \alpha} s^{\beta \alpha} \beta^{\beta \alpha+\alpha}}{\alpha^{2 \alpha+\beta \alpha} n^{\beta \alpha}}\right)^{s} .
$$

Reasoning as before, we obtain

$$
\log \left(r c e^{1-r c}\right)+(\beta \alpha+\alpha) \log \beta-(2 \alpha+\beta \alpha) \log \alpha+\alpha \log C^{\prime \prime} .
$$

The second term is negative, and among the others, for $K$ large enough, the first term dominates them in absolute value, and hence the expression is negative.

Finally, if $\alpha>1 / K^{1 / 4}$ and hence $\beta<K^{\prime} / K^{3 / 4}$, for some $C^{\prime \prime}>0$ we have

$$
2^{-\omega_{n}} \mathbb{E}\left(X_{u}^{s, \alpha s, n_{1}, n_{2}}\right) \leq\left(\frac{C^{\prime \prime \alpha} r c e^{1-r c} s^{\beta \alpha} \beta^{\beta \alpha+\alpha}}{\alpha^{2 \alpha+\beta \alpha} n^{\beta \alpha}}\right)^{s} .
$$

As before, we obtain

$$
\begin{array}{r}
\log \left(r c e^{1-r c}\right)+(\beta \alpha+\alpha) \log \beta-(2 \alpha+\beta \alpha) \log \alpha+\alpha \log C^{\prime \prime} \\
=\log \left(r c e^{1-r c}\right)+\beta \alpha \log (\beta / \alpha)+\alpha \log \left(C^{\prime \prime} \beta / \alpha^{2}\right) .
\end{array}
$$


Once more for $K$ large enough, $\beta<\alpha$, and the second term is negative. Also, for $K$ large enough, $C^{\prime \prime}<K^{1 / 4} / K^{\prime}$, and thus $C^{\prime \prime} \beta<1 / K^{1 / 2}$, and therefore $C^{\prime \prime} \beta<\alpha^{2}$, and the last expression is negative as well. In all cases, since we have $s=\Theta(u)$,

$$
2^{-\omega_{n}} \mathbb{E}\left(X_{u}^{s, \alpha s, n_{1}, n_{2}}\right) \leq \rho^{s} \leq \rho^{C u},
$$

for some $0<\rho<1$ and some absolute constant $C>0$. Clearly,

$$
\mathbb{E}\left(X_{u}\right)=\sum_{s, \alpha s, n_{1}, n_{2}} \mathbb{E}\left(X_{u}^{s, \alpha s, n_{1}, n_{2}}\right) \leq 2^{\omega_{n}} u^{4} \rho^{C u}
$$

Finally,

$$
\sum_{\log \log n \leq u \leq \epsilon n} \mathbb{E}\left(X_{u}\right) \leq 2^{\omega_{n}} \sum_{\log \log n \leq u \leq \epsilon n} u^{4} \rho^{C u}=o(1) .
$$

By Markov's inequality the lemma follows.

\section{ACKNOWLEDGEMENTS}

We thank Louis Theran for helpful comments regarding Remark 3.4 .

\section{REFERENCES}

[1] M. Ajtai, J. Komlós and E. Szemerédi. "The longest path in a random graph", Combinatorica, 1, 1-12 (1980).

[2] B. Bollobás. "Random graphs", Cambridge University Press, 2nd edition, 2001.

[3] W. Bresser, P. Boolchand, and P. Suranyi. "Rigidity Percolation and Molecular Clustering in Network Glasses", Phys. Rev. Lett. 56, 2493 (1986).

[4] P. M. Duxbury, D. J. Jacobs, M. F. Thorpe, and C. Moukarzel. "Floppy modes and the free energy: Rigidity and connectivity percolation on Bethe lattices", Phys. Rev. E 59, 2084 (1999).

[5] P. Gao, N. Wormald. "Orientability thresholds for random hypergraphs", Combinatorics, Probability and Computing, to appear.

[6] S. Janson and M. Luczak. "A simple solution to the $k$-core problem", Random Structures and Algorithms 30, 50-62 (2007).

[7] S. P. Kasiviswanathan, C. Moore and L. Theran. "The rigidity transition in random graphs", Proceedings of the twenty-second annual ACM-SIAM symposium on Discrete Algorithms (SODA) 2011, 1237-1252.

[8] G. Laman. "On graphs and rigidity of plane skeletal structures", J. Engrg. Math. 4, 331-340 (1970).

[9] Audrey Lee and Ileana Streinu. Pebble game algorithms and sparse graphs. Discrete Math., 308(8):1425-1437, 2008.

[10] A. Lee, I. Streinu and L. Theran "Graded Sparse Graphs and Matroids" Journal of Universal Computer Science 13, 1671-1679 (2007).

[11] M. Lelarge. "A new approach to the orientation of random hypergraphs", Proceedings of the twenty-third annual ACM-SIAM symposium on Discrete Algorithms (SODA) 2012, 251-264.

[12] M. Lelarge. "Diffusion and Cascading Behavior in Random Networks", Games Econ. Behav., 75(2):752-775 (2012).

[13] C. Moukarzel. "Rigidity percolation in a field", Phys. Rev. E 68, 056104 (2003).

[14] C. Moukarzel, P. M. Duxbury, and P. L. Leath. "First-order rigidity on Cayley trees", Phys. Rev. E 55, 5800 (1997).

[15] Jaroslav Nešetřil and Patrice Ossona de Mendez. Sparsity, volume 28 of Algorithms and Combinatorics. Springer, Heidelberg, 2012. Graphs, structures, and algorithms.

[16] J. C. Phillips and M. F. Thorpe. "Constraint theory, vector percolation and glass formation", Solid State Comm. 53, 699-702 (1985).

[17] A. J. Rader, B. M. Hespenheide, L. A. Kuhn, and M. F. Thorpe. "Protein unfolding: Rigidity lost" Proc. Nat. Acad. Sc. 99, 3540-3545 (2002).

[18] I. Streinu and L. Theran. "Slider-pinning rigidity: a maxwell-laman-type theorem", Discrete Comput. Geom. 44,812-837 (2010).

[19] L. Theran. "Rigid components of random graphs", Proceedings of CCCG'09 (2009). 
[20] M. F. Thorpe. "Continuous deformations in random networks", J. Non-Cryst. Sol. 57, 355370 (1983). 